\title{
Brain dysfunction in chronic pain patients assessed by resting-state electroencephalography
}

\author{
Son Ta Dinh ${ }^{a, b}$, Moritz M. Nickel ${ }^{a, b}$, Laura Tiemann ${ }^{a, b}$, Elisabeth S. May ${ }^{a, b}$, Henrik Heitmann ${ }^{a, b}$, Vanessa D. Hohn ${ }^{a, b}$,
} Günther Edenharter ${ }^{\mathrm{c}}$, Daniel Utpadel-Fischler ${ }^{\mathrm{a}}$, Thomas R. Tölle ${ }^{\mathrm{a}}$, Paul Sauseng ${ }^{\mathrm{d}}$, Joachim Gross ${ }^{\mathrm{e}, \mathrm{f}}$, Markus Ploner ${ }^{\mathrm{a}, \mathrm{b}, *}$

\begin{abstract}
Chronic pain is a common and severely disabling disease whose treatment is often unsatisfactory. Insights into the brain mechanisms of chronic pain promise to advance the understanding of the underlying pathophysiology and might help to develop disease markers and novel treatments. Here, we systematically exploited the potential of electroencephalography to determine abnormalities of brain function during the resting state in chronic pain. To this end, we performed state-of-the-art analyses of oscillatory brain activity, brain connectivity, and brain networks in 101 patients of either sex suffering from chronic pain. The results show that global and local measures of brain activity did not differ between chronic pain patients and a healthy control group. However, we observed significantly increased connectivity at theta $(4-8 \mathrm{~Hz})$ and gamma $(>60 \mathrm{~Hz})$ frequencies in frontal brain areas as well as global network reorganization at gamma frequencies in chronic pain patients. Furthermore, a machine learning algorithm could differentiate between patients and healthy controls with an above-chance accuracy of 57\%, mostly based on frontal connectivity. These results suggest that increased theta and gamma synchrony in frontal brain areas are involved in the pathophysiology of chronic pain. Although substantial challenges concerning the reproducibility of the findings and the accuracy, specificity, and validity of potential electroencephalography-based disease markers remain to be overcome, our study indicates that abnormal frontal synchrony at theta and gamma frequencies might be promising targets for noninvasive brain stimulation and/or neurofeedback approaches.
\end{abstract}

Keywords: Chronic pain, Electroencephalography, Oscillations, Synchrony, Brain networks, Theta, Gamma

\section{Introduction}

Chronic pain is a disease characterized by ongoing pain and significant sensory, cognitive, and affective abnormalities ${ }^{49,77}$ that have detrimental effects on quality of life. Affecting between $20 \%$ and $30 \%$ of the adult population, ${ }^{8,35}$ chronic pain is a leading cause of disability worldwide. ${ }^{27}$ Thus, advances in the understanding and treatment of chronic pain are urgently needed.

Studies in animals and humans have revealed that chronic pain is associated with significant structural and functional changes of

\footnotetext{
Sponsorships or competing interests that may be relevant to content are disclosed at the end of this article.

a Department of Neurology, School of Medicine, Technical University of Munich, Munich, Germany, ${ }^{b}$ TUM-Neuroimaging Center, School of Medicine, Technical University of Munich, Munich, Germany, ${ }^{\circ}$ Department of Anesthesiology, School of Medicine, Technical University of Munich, Munich, Germany, ${ }^{d}$ Department of Psychology, Ludwig-Maximilians-Universität München, Munich, Germany, ${ }^{e}$ Institute for Biomagnetism and Biosignalanalysis, University of Münster, Münster, Germany, ${ }^{f}$ Centre for Cognitive Neuroimaging, University of Glasgow, Glasgow, United Kingdom

${ }^{*}$ Corresponding author. Address: Department of Neurology, School of Medicine, Technical University of Munich, Ismaninger Str 22, 81675 Munich, Germany. Tel.: +49-89-4140-4608; fax: +49-89-4140-4867. E-mail address: markus.ploner@tum.de (M.Ploner).

PAIN 160 (2019) 2751-2765
}

Copyright (C) 2020 The Author(s). Published by Wolters Kluwer Health, Inc. on behalf of the International Association for the Study of Pain. This is an open access article distributed under the Creative Commons Attribution License 4.0 (CCBY), which permits unrestricted use, distribution, and reproduction in any medium, provided the original work is properly cited.

http://dx.doi.org/10.1097/j.pain.0000000000001666 the brain. ${ }^{2,38,55}$ In particular, the prefrontal cortex and subcortical brain areas including amygdala, hippocampus, and striatal areas have been implicated in chronic pain. 2,3,31,47,55,62,72 Further insights into the brain mechanisms of chronic pain not only promise to advance the understanding of the underlying pathophysiology but could also be clinically highly useful. In particular, a brain-based marker of chronic pain could be immensely helpful for the diagnosis, prognosis, classification, prevention, and treatment of pain. ${ }^{15,71}$ Accordingly, the feasibility, limitations, and perspectives of brain-based markers of pain are currently intensively discussed in the pain research community $^{15,50,64}$ and beyond. ${ }^{43,56,84}$ Recent functional magnetic resonance imaging (fMRI) studies have made important first steps towards such a brain-based marker of experimental ${ }^{80}$ and chronic pain. ${ }^{44,45}$

Using electroencephalography (EEG) to assess abnormalities of brain function and to establish a brain-based marker of chronic pain is particularly appealing because it is safe, cost-effective, broadly available, and potentially mobile. Moreover, an EEGbased marker of chronic pain might not only be helpful for the diagnosis and classification of chronic pain but might itself represent a target for novel therapeutic strategies such as neurofeedback ${ }^{63}$ or noninvasive brain stimulation techniques. ${ }^{54}$ As a potential first step in that direction, some EEG studies have shown a slowing of neural oscillations together with an increase of oscillatory brain activity at theta frequencies (4-8 Hz) in chronic pain patients. ${ }^{58,76}$ These observations have been embedded in the thalamocortical dysrhythmia (TCD) model of neuropsychiatric disorders. ${ }^{41}$ In this model, abnormal thalamocortical theta activity 
yields abnormal oscillations at gamma $(>30 \mathrm{~Hz})$ frequencies, which eventually result in different neuropsychiatric symptoms including ongoing pain. This model is highly appealing, but evidence is sparse, contradictory, and mostly confined to small groups of patients suffering from neuropathic subtypes of chronic pain. ${ }^{52,53}$ Thus, a general EEG-based marker of chronic pain remains to be demonstrated.

In this study, we aimed to systematically and extensively exploit the potential of EEG to determine abnormalities of resting-state brain activity as a potential brain-based marker of chronic pain. In a large cohort of chronic pain patients and age- and sex-matched healthy controls, we analyzed global and local measures of oscillatory brain activity. Moreover, we performed connectivity analyses using phasebased and amplitude-based measures in source space as well as graph theory-based network analyses. In a univariate approach, we statistically compared these measures between patients and healthy controls. Moreover, in a multivariate machine learning approach, we tested whether patterns of these measures allow to distinguish between chronic pain patients and healthy controls.

\section{Materials and methods}

\subsection{Participants}

One hundred one patients (age $58.2 \pm 13.5$ years [mean $\pm \mathrm{SD}$ ], 69 female) suffering from chronic pain and 84 age- and sex-matched healthy control participants (age $57.8 \pm 14.6$ years, 55 female) participated in the study. Inclusion criteria for patients were a clinical diagnosis of chronic pain, with pain lasting at least 6 months and a minimum reported average pain intensity $\geq 4 / 10$ during the past 4 weeks ( $0=$ no pain, $10=$ worst imaginable pain). Exclusion criteria were acute changes of the pain condition during the past 3 months, for example, due to recent injuries or surgeries. Further exclusion criteria were major neurological diseases such as stroke, epilepsy, or dementia, major psychiatric diseases aside from depression, and severe general diseases. Finally, patients on medication with benzodiazepines were excluded, other medication was not restricted, and patients' medication was maintained. Demographic and clinical details of participants are shown in Tables 1 and 2, respectively. In summary, we included 47 patients with chronic back pain, 30 patients with chronic widespread pain, 6 patients with joint pain, 5 patients with unspecific neuropathic pain, 7 patients with postherpetic neuralgia, and 6 patients with polyneuropathic pain. All participants provided written informed

\section{Table 1}

Demographics and questionnaire results.

\begin{tabular}{lll}
\hline & $\begin{array}{l}\text { CP (N = 101, } \\
\text { mean } \pm \mathbf{~ S D )}\end{array}$ & $\begin{array}{l}\text { HC (N = 84, } \\
\text { mean } \pm \mathbf{~ S D )}\end{array}$ \\
\hline Gender (M/F) & $32 / 69$ & $29 / 55$ \\
\hline Age & $58.2 \pm 13.5$ & $57.8 \pm 14.6$ \\
\hline VR-12 PCS score & $31.7 \pm 7.8$ & $52.7 \pm 5.7$ \\
\hline VR-12 MCS score & $46.4 \pm 12.0$ & $54.1 \pm 9.0$ \\
\hline Current pain intensity (VAS) & $5.2 \pm 1.9$ & - \\
\hline Avg. Pain intensity (VAS) & $5.7 \pm 1.6$ & - \\
\hline Pain duration (mo) & $121.9 \pm 114.3$ & - \\
\hline PDI score & $28.3 \pm 14.7$ & - \\
\hline PDQ score & $17.4 \pm 6.5$ & - \\
\hline Avg. pain intensity, average pain intensity in the past 4 weeks; CP, chronic pain patients; HC, healthy controls; \\
MCS, mental component score; PCS, physical component score; PDI, pain disability index; PDQ, \\
painDETECT; VAS, visual analogue scale; VR-12, Veteran's RAND 12.
\end{tabular}

consent. The study was approved by the ethics committee of the Medical Faculty of the Technical University of Munich and conducted in accordance with the relevant guidelines and regulations. A power analysis for independent 2-sample $t$-tests using $\mathrm{G}^{*} \mathrm{Power}^{22}$ showed that the number of participants allowed for detecting differences between groups of at least medium effect size (Cohen's $d=0.5$ ) with a statistical power of 0.9.

\subsection{Recordings}

Brain activity was recorded using EEG. Recordings were performed during the resting state, ie, participants were asked to stay in a relaxed and wakeful state, without any particular task. Electroencephalography data were recorded with eyes closed and eyes open for 5 minutes each. Because the eyes-closed condition showed better data quality and less muscle artifacts, analyses were focused on this condition.

Electroencephalography data were recorded using 64 electrodes consisting of all 10-20 system electrodes and the additional electrodes Fpz, CPz, POz, Oz, Iz, AF3/4, F5/6, FC1/2/3/4/5/6, FT7/8/9/10, C1/2/5/6, CP1/2/3/4/5/6, TP7/8/9/10, P5/6, and P01/2/9/10 plus 2 electrodes below the outer canthus of each eye (Easycap, Herrsching, Germany) and BrainAmp MR plus amplifiers (Brain Products, Munich, Germany). All EEG electrodes were referenced to FCz and grounded at AFz. Simultaneously, muscle activity was recorded with 2 bipolar surface electromyography (EMG) electrode montages placed on the right masseter and neck (semispinalis capitis and splenius capitis) muscles ${ }^{14}$ and a BrainAmp ExG MR amplifier (Brain Products). The EMG ground electrode was placed at vertebra C2. All data were sampled at $1000 \mathrm{~Hz}(0.1 \mu \mathrm{V}$ resolution) and band-pass filtered between 0.016 and $250 \mathrm{~Hz}$. Impedances were kept below $20 \mathrm{k} \Omega$.

Before the EEG recordings, patients completed the following questionnaires to assess pain characteristics and comorbidities: short-form McGill pain Questionnaire, ${ }^{48}$ Pain Disability Index, ${ }^{18}$ painDETECT (PDQ), ${ }^{24}$ Beck Depression Inventory ॥ (BDI-II), ${ }^{6}$ State-Trait-Anxiety Inventory, ${ }^{65}$ and the Veteran's RAND 12-Item Health Survey (VR-12). ${ }^{61}$

\subsection{Preprocessing}

Preprocessing was performed using the BrainVision Analyzer software (Brain Products). Data were downsampled to $250 \mathrm{~Hz}$. For artifact identification, a high-pass filter of $1 \mathrm{~Hz}$ and a $50-\mathrm{Hz}$ notch filter for line noise removal were applied to the EEG data. Independent component analysis was performed, ${ }^{34}$ and components representing eye movements and muscle artifacts were identified based on time courses and topographies. Furthermore, time intervals of $400 \mathrm{~ms}$ around data points with signal jumps exceeding $\pm 100 \mu \mathrm{V}$ were marked for rejection. Finally, all data were visually inspected and remaining bad segments marked. Subsequently, independent components representing artifacts were subtracted from the raw, unfiltered EEG data ${ }^{83}$ and EEG data were re-referenced to the average reference. The reference electrode FCz was added to the signal array.

Next, data were exported from the BrainVision Analyzer and further analyzed in Matlab (Mathworks, Natick, MA) with the FieldTrip ${ }^{51}$ and Brain Connectivity Toolbox, ${ }^{57}$ along with customwritten code. Data were segmented into 2-second epochs with 1-second overlap. A 2-second epoch length was chosen to balance the stationarity of the signals and the number of samples for lower frequencies (down to $4 \mathrm{~Hz}$ ). ${ }^{11,73}$ All analyses are based on these epochs and the following 4 frequency bands: theta (4-8 $\mathrm{Hz})$, alpha (8-13 Hz), beta (14-30 Hz), and gamma $(60-100 \mathrm{~Hz})$. 
Table 2

\section{Patient characteristics.}

\begin{tabular}{|c|c|c|c|c|c|c|c|c|c|}
\hline ID & Age (y) & Sex & Pain duration (mo) & Curr. Pain (VAS) & Avg. Pain (VAS) & Pain diagnosis & MQS & BDI & PDQ \\
\hline 1 & 67 & $\mathrm{~m}$ & 360 & 5 & 5 & CBP & 4 & 3 & 30 \\
\hline 2 & 54 & $f$ & 120 & 4 & 5 & CBP & 32 & 35 & 14 \\
\hline 3 & 64 & $f$ & 96 & 5 & 7 & CBP & 11 & 7 & 16 \\
\hline 4 & 41 & $\mathrm{~m}$ & 24 & 7 & 6 & CBP & 6 & 15 & 22 \\
\hline 5 & 74 & $\mathrm{~m}$ & 180 & 3 & 5 & CBP & 7 & 18 & 4 \\
\hline 6 & 58 & $f$ & 168 & 7 & 6 & CBP & 19 & 22 & 21 \\
\hline 7 & 65 & $\mathrm{~m}$ & 48 & 6 & 5 & CBP & 4 & 11 & 12 \\
\hline 8 & 65 & $f$ & 132 & 3 & 3 & CBP & 6 & 5 & 24 \\
\hline 9 & 76 & $f$ & 24 & 5 & 5 & CBP & 4 & 9 & 20 \\
\hline 10 & 33 & $f$ & 36 & 6 & 7 & CBP & 9 & 19 & 16 \\
\hline 11 & 45 & $f$ & 12 & 8 & 7 & CBP & 5 & 20 & 8 \\
\hline 12 & 51 & $f$ & 24 & 5 & 6 & CBP & 11 & 22 & 19 \\
\hline 13 & 73 & $f$ & 108 & 6 & 8 & CBP & 11 & 21 & 26 \\
\hline 14 & 41 & $f$ & 120 & 5 & 6 & CBP & 13 & 14 & 13 \\
\hline 15 & 55 & $f$ & 252 & 9 & 9 & CBP & 24 & 24 & 20 \\
\hline 16 & 73 & $\mathrm{~m}$ & 300 & 5 & 4 & CBP & 4 & 10 & 5 \\
\hline 17 & 46 & $\mathrm{~m}$ & 360 & 6 & 7 & CBP & 16 & 10 & 5 \\
\hline 18 & 50 & $\mathrm{~m}$ & 60 & 7 & 7 & CBP & 0 & 5 & 17 \\
\hline 19 & 59 & $f$ & 24 & 4 & 6 & CBP & 12 & 31 & 23 \\
\hline 20 & 62 & $\mathrm{~m}$ & 12 & 5.5 & 6 & CBP & 10 & 26 & 22 \\
\hline 21 & 54 & $\mathrm{~m}$ & 84 & 5 & 7 & CBP & 16 & 18 & 17 \\
\hline 22 & 39 & $f$ & 144 & 4 & 2 & CBP & 0 & 12 & 22 \\
\hline 23 & 66 & $\mathrm{~m}$ & 48 & 4 & 4 & CBP & 8 & 19 & 10 \\
\hline 24 & 57 & $\mathrm{~m}$ & 300 & 5 & 6 & CBP & 22 & 12 & 17 \\
\hline 25 & 52 & $\mathrm{~m}$ & 300 & 4 & 3 & CBP & 11 & 22 & 13 \\
\hline 26 & 47 & $f$ & 180 & 4 & 3.5 & CBP & 26 & 19 & 15 \\
\hline 27 & 24 & $f$ & 96 & 7.5 & 7.5 & CBP & 5 & 13 & 20 \\
\hline 28 & 59 & f & 180 & 3 & 5 & CBP & 6 & 4 & 10 \\
\hline 29 & 82 & $\mathrm{~m}$ & 60 & 5 & 5 & CBP & 14 & 21 & 8 \\
\hline 30 & 54 & $\mathrm{~m}$ & 120 & 6.5 & 6 & CBP & 12 & 17 & 22 \\
\hline 31 & 62 & $f$ & 36 & 3 & 4.5 & CBP & 3 & 22 & 11 \\
\hline 32 & 73 & $\mathrm{~m}$ & 480 & 8 & 8 & CBP & 4 & 11 & 10 \\
\hline 33 & 48 & $\mathrm{~m}$ & 64 & 0.5 & 4 & CBP & 24.45 & 24 & 22 \\
\hline 34 & 55 & $f$ & 240 & 5 & 6 & CBP & 8.55 & 31 & 11 \\
\hline 35 & 59 & $f$ & 60 & 3 & 4 & PNP & 13.5 & 11 & 15 \\
\hline 36 & 48 & $f$ & 108 & 3 & 4 & PHN & 15.4 & 13 & 28 \\
\hline 37 & 73 & $f$ & 24 & 5 & 5 & CBP & 13.5 & 5 & 11 \\
\hline 38 & 57 & $\mathrm{~m}$ & 84 & 8 & 7.5 & PNP & 22.35 & 15 & 33 \\
\hline 39 & 57 & $f$ & 96 & 6 & 7 & CBP & 3.4 & 20 & 15 \\
\hline 40 & 77 & $f$ & 36 & 5 & 7.8 & PNP & 4 & 10 & 11 \\
\hline 41 & 77 & $f$ & 480 & 3.5 & 3.5 & CBP & 8.6 & 9 & 19 \\
\hline 42 & 53 & $f$ & 24 & 4 & 5 & CBP & 16.3 & 12 & 24 \\
\hline 43 & 80 & $\mathrm{~m}$ & 48 & 4 & 4 & PHN & 11.4 & 4 & 18 \\
\hline 44 & 77 & $f$ & 192 & 4 & 6 & CWP & 12.8 & 0 & 23 \\
\hline 45 & 57 & $f$ & 72 & 5 & 6 & CWP & 5.7 & 42 & 17 \\
\hline
\end{tabular}


Table 2 (continued)

\begin{tabular}{|c|c|c|c|c|c|c|c|c|c|}
\hline ID & Age (y) & Sex & Pain duration (mo) & Curr. Pain (VAS) & Avg. Pain (VAS) & Pain diagnosis & MQS & BDI & PDQ \\
\hline 46 & 67 & $f$ & 21 & 6 & 8 & NP & 3.4 & 8 & 17 \\
\hline 47 & 77 & $f$ & 324 & 6 & 6 & CWP & 3.4 & 16 & 24 \\
\hline 48 & 61 & $f$ & 36 & 4.5 & 6 & NP & 3.4 & 7 & 21.5 \\
\hline 49 & 65 & $f$ & 23 & 8 & 8 & CWP & 4 & 7.5 & 25 \\
\hline 50 & 65 & $f$ & 180 & 5 & 4 & CWP & 0 & 12 & 22 \\
\hline 51 & 56 & $f$ & 213 & 4 & 7 & CWP & 8 & 13 & 23 \\
\hline 52 & 57 & $\mathrm{~m}$ & 264 & 3 & 4 & $J P$ & 13.25 & 10 & 11 \\
\hline 53 & 41 & $f$ & 24 & 4 & 5 & NP & 25.5 & 12 & 24 \\
\hline 54 & 69 & $f$ & 72 & 8 & 5 & CBP & 0 & 19 & 13 \\
\hline 55 & 56 & $f$ & 108 & 8 & 8 & PNP & 17.2 & 10 & 25 \\
\hline 56 & 72 & $f$ & 120 & 7 & 8 & CWP & 15.4 & 16 & 20 \\
\hline 57 & 57 & $\mathrm{~m}$ & 96 & 5 & 7 & PNP & 10.9 & 11 & 28 \\
\hline 58 & 82 & $f$ & 36 & 2 & 5 & CBP & 4.6 & n.a. & 10 \\
\hline 59 & 70 & $f$ & 420 & 4 & 6 & CWP & 14.8 & 28 & 22 \\
\hline 60 & 70 & $f$ & 72 & 4 & 6 & PHN & 10.3 & 11 & 22 \\
\hline 61 & 54 & $f$ & 24 & 7 & 5 & PHN & 5.8 & 10 & 10 \\
\hline 62 & 69 & $f$ & 48 & 6 & 5 & PHN & 8.4 & 6 & 16 \\
\hline 63 & 66 & $f$ & 84 & 2 & 3 & $\mathrm{JP}$ & 6 & 4 & 13 \\
\hline 64 & 52 & $f$ & 204 & 6 & 6 & CWP & 10.2 & 16 & 21 \\
\hline 65 & 77 & $f$ & 72 & 8 & 8 & $J P$ & 4.6 & n.a. & 19 \\
\hline 66 & 42 & $\mathrm{~m}$ & 252 & 7 & 9 & CBP & 17.35 & 33 & 22 \\
\hline 67 & 51 & $f$ & 31 & 6 & 5 & $\mathrm{JP}$ & 9.1 & 15 & 7 \\
\hline 68 & 55 & $\mathrm{~m}$ & 7 & 7 & 7 & $\mathrm{JP}$ & 6.9 & 1 & 13 \\
\hline 69 & 68 & $f$ & 24 & 1 & 4 & NP & 3.8 & 0 & 23 \\
\hline 70 & 71 & $\mathrm{~m}$ & 324 & 4 & 5 & CBP & 10.8 & 5 & 6 \\
\hline 71 & 24 & $f$ & 108 & 6 & 6 & CWP & 8 & 31 & 21 \\
\hline 72 & 71 & $\mathrm{~m}$ & 36 & 6 & 6 & CBP & 5.8 & 9 & 14 \\
\hline 73 & 68 & $f$ & 204 & 3 & 4 & $\mathrm{JP}$ & 8.8 & 7 & 15 \\
\hline 74 & 86 & $f$ & 120 & 3 & 3 & CBP & 2 & 5 & 6 \\
\hline 75 & 68 & $f$ & 120 & 4 & 5 & NP & 7.8 & 5 & 28 \\
\hline 76 & 45 & $\mathrm{~m}$ & 48 & 1 & 2 & CBP & 3.8 & 10 & 9 \\
\hline 77 & 18 & $f$ & 16 & 4 & 6 & PHN & 0 & 14 & 20 \\
\hline 78 & 80 & $f$ & 25 & 8 & 7 & PHN & 23.1 & 15 & 20 \\
\hline 79 & 60 & $\mathrm{~m}$ & 60 & n.a. & n.a. & PNP & 0 & n.a. & n.a. \\
\hline 80 & 60 & $f$ & 54 & 3.5 & 5.5 & CBP & 13.5 & 22 & 18 \\
\hline 81 & 57 & $\mathrm{~m}$ & 17 & 6.5 & 6.5 & CBP & 24.3 & 25 & 24 \\
\hline 82 & 45 & $\mathrm{~m}$ & n.a. & 7.3 & n.a. & CWP & 0 & 17 & n.a. \\
\hline 83 & 24 & $f$ & n.a. & 3.2 & n.a. & CWP & 0 & 17 & n.a. \\
\hline 84 & 49 & $\mathrm{~m}$ & n.a. & 7.3 & n.a. & CWP & 0 & 39 & n.a. \\
\hline 85 & 47 & $f$ & n.a. & 2.4 & n.a. & CWP & 0 & 21 & n.a. \\
\hline 86 & 53 & $f$ & n.a. & 7.5 & n.a. & CWP & 0 & 22 & n.a. \\
\hline 87 & 41 & $f$ & n.a. & 6.2 & n.a. & CWP & 0 & 29 & n.a. \\
\hline 88 & 46 & $\mathrm{~m}$ & n.a. & 7.4 & n.a. & CWP & 0 & 20 & n.a. \\
\hline 89 & 56 & $f$ & n.a. & 1.8 & n.a. & CWP & 0 & 22 & n.a. \\
\hline 90 & 55 & $f$ & n.a. & 7.8 & n.a. & CWP & 0 & 30 & n.a. \\
\hline 91 & 60 & $f$ & n.a. & 6.2 & n.a. & CWP & 0 & 14 & n.a. \\
\hline
\end{tabular}


Table 2 (continued)

\begin{tabular}{|c|c|c|c|c|c|c|c|c|c|}
\hline ID & Age (y) & Sex & Pain duration (mo) & Curr. Pain (VAS) & Avg. Pain (VAS) & Pain diagnosis & MQS & BDI & PDQ \\
\hline 92 & 55 & $f$ & n.a. & 8.6 & n.a. & CWP & 0 & 25 & n.a. \\
\hline 93 & 48 & $f$ & n.a. & 2.9 & n.a. & CWP & 0 & 27 & n.a. \\
\hline 94 & 59 & $\mathrm{~m}$ & n.a. & 6.8 & n.a. & CWP & 0 & 13 & n.a. \\
\hline 95 & 60 & $f$ & n.a. & 5.0 & n.a. & CWP & 0 & 17 & n.a. \\
\hline 96 & 58 & $f$ & n.a. & 4.5 & n.a. & CWP & 0 & 12 & n.a. \\
\hline 97 & 71 & $f$ & n.a. & 8.4 & n.a. & CWP & 0 & 36 & n.a. \\
\hline 98 & 42 & $\mathrm{~m}$ & n.a. & 3.7 & n.a. & CWP & 0 & 12 & n.a. \\
\hline 99 & 38 & $f$ & n.a. & 6.5 & n.a. & CWP & 0 & 10 & n.a. \\
\hline 100 & 65 & $f$ & n.a. & 8.8 & n.a. & CWP & 0 & 18 & n.a. \\
\hline 101 & 60 & $f$ & n.a. & 6.0 & п.а. & CWP & 0 & 21 & п.а. \\
\hline
\end{tabular}

Avg. pain, average pain in the past 4 weeks; BDI, Beck Depression Inventory II, score $\geq 18$ = clinically relevant depression; CBP, chronic back pain; Curr. pain, currently experienced pain; CWP, chronic widespread pain; $\mathrm{f}$, female; ID, patient identification number; JP, joint pain; $\mathrm{m}$, male; MQS, medication quantification scale; n.a., not available because the respective questionnaire was not completed; NP, neuropathic pain; PDQ, painDETECT, score $\geq 19$ = neuropathic pain component probable; PHN, postherpetic neuralgia; PNP, polyneuropathic pain; VAS, visual analogue scale: $0=$ no pain, $10=$ worst imaginable pain.

We observed strong nonstationary line noise around $45-55 \mathrm{~Hz}$ and therefore excluded the "low gamma" band from frequency band-specific analyses.

\subsection{Electroencephalography analysis-overview}

Figure 1 summarizes the analyses of the EEG data. The analyses included measures of oscillatory brain activity (power) and connectivity in electrode and source space, respectively. Two categories of analyses were performed. First, local, ie, spatially specific, analyses were performed in which a single value is obtained for every electrode, voxel, or brain region. These analyses included comparisons of power topographies on electrode level and comparisons of connectivity and local network measures topographies (degree and clustering coefficient [CC]) on source level. Second, global, ie, spatially holistic, analyses were performed. These analyses include all analyses that average across all electrodes, voxels, or brain regions, ie, the peak frequency, the power spectrum, and all global network measures (see below). In all global analyses, each participant is represented by a single scalar value per measure. All analyses were based on 2-second epochs of resting-state data to balance robustness, frequency resolution, and nonstationarity of the data.

\subsection{Brain activity (power) analysis}

Oscillatory brain activity (power) was analyzed in electrode space. Frequency-specific global power was computed for all epochs using a Fast Fourier Transformation with Slepian multitapers ${ }^{68}$ and a frequency smoothing of $1 \mathrm{~Hz}$ and then averaged across epochs and electrodes. Power was first computed for the complete power spectrum, ie, 1 to $100 \mathrm{~Hz}$, with a frequency resolution of $0.5 \mathrm{~Hz}$. To remove line noise, a band-stop filter of 45 to $55 \mathrm{~Hz}$ was applied before computing power.

The individual dominant peak frequency was determined on the average of the epochs as the highest local maximum (larger than its 2 neighboring samples) of the amplitude in the frequency range of 6 to $14 \mathrm{~Hz} .{ }^{5}$ We also pursued alternative approaches to determine the peak frequency by (1) computing the center of gravity of the power spectrum, ${ }^{5,36}$ (2) computing the dominant peak frequency using longer time windows of 5 seconds, and by (3) computing the peak frequency on each single epoch and then averaging the peaks. ${ }^{26}$
To compare the spatial distribution of local brain activity between patients and healthy controls, power was averaged within each frequency band before comparing frequency-specific power topographies between groups using cluster-based permutation tests.

Relative power was calculated by normalizing every power value (both local and global power) by the respective participant's total power. Total power was calculated by summing all power values across frequencies from 1 to $100 \mathrm{~Hz}$ and across all electrodes.

\subsection{Connectivity analysis}

Connectivity analyses were performed in source space. For source analysis, we used linearly constrained minimum variance beamforming ${ }^{74}$ to project the band-pass filtered data for each frequency band and participant from electrode space into source space. This was done with a combination of the FieldTrip toolbox ${ }^{51}$ and custom-written code. Spatial filters for every single person were computed based on the covariance matrices of the band-pass filtered data for each frequency band and a lead field matrix. A 3-dimensional grid with a 1-cm resolution covering the brain was defined, resulting in a total of 2020 voxels in the brain. The lead field was constructed for each voxel using a realistically shaped 3-shell boundary-element volume conduction model based on the template Montreal Neurological Institute (MNI) brain. We used a regularization parameter of $5 \%$ of the covariance matrix and chose the dipole orientation of most variance using singular value decomposition. Finally, the preprocessed and band-pass filtered EEG data of each subject were projected through the individual spatial filter to extract the amplitude time series of neuronal activity of each frequency band at each voxel.

Connectivity analyses of EEG data were performed using phase-based and amplitude-based approaches that likely capture different and complementary neural communication processes (see Ref. 20 for a review of these approaches and communication processes). In brief, amplitude-based connectivity is believed to be more closely related to structural connectivity and putatively regulates activation of brain regions. ${ }^{20}$ By contrast, phase-based connectivity seems more detached from structure and more strongly affected by contextual factors. ${ }^{20}$ Here, functional connectivity was investigated using the phase locking value (PLV), ${ }^{40}$ the debiased weighted phase lag 


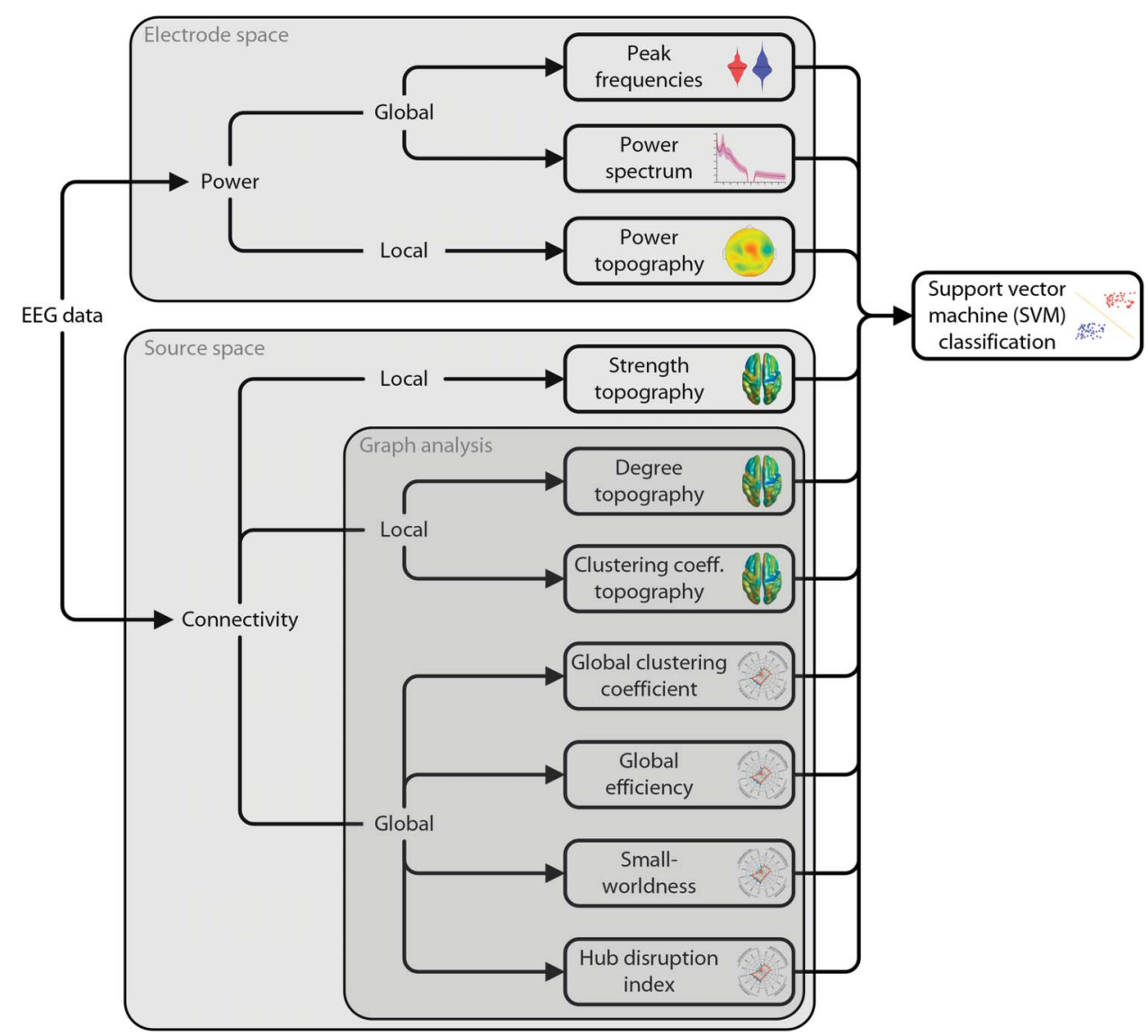

Figure 1. Analysis pipeline. Electroencephalography data were analyzed with regards to power and connectivity, which quantify neural activity and neural communication, respectively. Power analyses were performed in electrode space. Analyses of functional connectivity were performed in source space. Connectivity analyses comprised phase-based (PLV, dwPLI) and amplitude-based (AEC) connectivity measures. Graph-theoretical network analysis was applied to further characterize functional connectivity. All measures were compared between chronic pain patients and healthy controls. In addition, a purely data-driven machine learning approach was adopted, using SVMs. The SVM was trained on all power and connectivity measures to distinguish between chronic pain patients and healthy controls. dwPLI, debiased weighted phase lag index; PLV, phase locking value; SVM, support vector machine.

index $(d w P L I),{ }^{78}$ and the orthogonalized amplitude envelope correlation (AEC). ${ }^{32}$ The PLV and dwPLI are based on the phase of the signals, whereas the AEC is based on the amplitude. The PLV is well established, highly sensitive, and captures both zero phase lag and nonzero phase lag connectivity. The PLV is sensitive to volume conduction effects because volume conduction can yield spurious synchrony at zero phase lag. Source space analysis can significantly reduce these volume conduction effects on PLV-based connectivity. ${ }^{29}$ Moreover, group contrasts of connectivity further reduce the likelihood of volume conduction effects because these effects are unlikely to differ between groups. The dwPLI captures nonzero phase lag connectivity only. The dwPLI is therefore not susceptible to volume conduction at the cost of reduced sensitivity because real synchrony at zero phase lag is also discarded. This similarly applies to connectivity analyses in electrode space and source space. Because of the explorative character of our study, we mainly report the more sensitive measure, the PLV.

For the connectivity analyses, the connectivity between every pair of voxels was computed, resulting in a $2020 \times 2020$ connectivity matrix, with a single value representing the strength of connection between 2 voxels over the complete recording time. All 3 connectivity measures are undirected.

\subsection{Graph-theoretical network analysis}

By applying graph-theoretical methods to the data, we reduced the high-dimensional EEG data to a few network measures, characterizing the organization of the whole brain network. Graph theory defines networks as collections of nodes and edges connecting the nodes to each other. We defined the nodes as voxels and the edges as thresholded functional connectivity between voxels. The connectivity matrix was thresholded to the 10\% (5\%, 20\%) strongest connections and binarized. A binary connectivity matrix was computed to reduce the computational load and to facilitate interpretation ${ }^{23}$ and comparison with previous connectivity analyses.

We used common graph measures that characterize either a single node (local analyses) or the complete network (global analyses). ${ }^{57}$ We investigated 2 local network measures: the degree and the local CC. The degree is the number of a node's edges, ie, the number of connections to other nodes. The local CC is the fraction of the node's neighbors that are also neighbors of each other. Thus, both local measures depict how well connected a node is. In particular, the degree indicates how well connected a node is to all other nodes of the network, and the local CC indicates how well connected a node is to neighboring nodes. Four global network measures were included in the analysis: the global CC (gCC), global efficiency (gEff), small-worldness (S), and hub disruption index $\left(\mathrm{k}_{\mathrm{d}}\right){ }^{1}$ The $\mathrm{gCC}$ is the average of the local CC of all nodes indicating the prevalence of clustered connectivity around individual nodes. ${ }^{57}$ In 
functional brain networks, it is commonly regarded as a measure of functional segregation. ${ }^{57}$ The global efficiency is the inverse of the average shortest path length. In functional brain networks, it represents a measure of functional integration. ${ }^{57}$ Small-worldness describes the ratio of $\mathrm{CC}$ and global efficiency and compares it with random networks. In functional brain networks, it can quantify the balance of functional segregation and integration ${ }^{57}$ and has been associated with the overall efficiency of communication in a network. ${ }^{82}$ Finally, the hub disruption index compares the degree of all nodes with those of a control group. Positive values indicate that strongly connected nodes increase and weakly connected nodes decrease their number of connections ("the rich get richer and the poor get poorer"). Conversely, negative values indicate that strongly connected nodes decrease and weakly connected nodes increase their number of connections ("the rich get poorer and the poor get richer"), which means a shift of the network towards a random network with less internal structure.

\subsection{Correlation analysis}

Pearson's $r$ was computed between clinical parameters and brain measures, which were found to show significant relationships either in previous studies ${ }^{10,17,21,28,39,45,58,59,67,75}$ or our own. The global peak frequency, mean global power in the 4 frequency bands, hub disruption index and the mean theta and gamma PLV connectivity (averaged across voxels of clusters with significant differences between patients and controls), the PLV global efficiency in the gamma band, and the dwPLI hub disruption index in the gamma band were correlated with the following clinical parameters: current pain intensity, average pain intensity in the past 4 weeks quantified by a visual analogue scale, pain duration, pain disability quantified by the pain disability index, ${ }^{18}$ mental and physical quality of life quantified by the VR$12,{ }^{61}$ depression quantified by the BDI-II, ${ }^{6}$ and medication as quantified by the medication quantification scale. ${ }^{30}$

\subsection{Machine learning analysis}

The machine learning analysis was conducted using the Statistics and Machine Learning Toolbox in Matlab as well as custom-written scripts. We implemented a support vector machine $(S V M)^{13}$ with a linear kernel, which was trained on all available features. The features were the peak frequency (one feature per participant), global power spectrum (199 features per participant, one feature for each frequency step), local power ( $4 \times 65$ features per participant, 65 electrodes), local strength of connectivity $(3 \times 4 \times 2020$ features per participant, 2020 voxels in 4 frequency bands and 3 connectivity measures), local degree $(3 \times 4 \times 2020$ features per participant, 2020 voxels in 4 frequency bands and 3 connectivity measures), local CC ( $3 \times 4 \times 2020$ features per participant, 2020 voxels in 4 frequency bands and 3 connectivity measures), and the global graph measures $(3 \times$ $4 \times 4$ features per participant, 4 global graph measures in 4 frequency bands and 3 connectivity measures). This resulted in an SVM containing 73,228 features per participant.

To avoid overfitting, we implemented a so-called sequential forward feature selection. This approach first tests the predictive value of all single features separately. It next takes the most predictive feature and adds a second feature and tests whether the classification improves. This procedure is repeated until no further improvements of the classification can be achieved. In our data, the procedure resulted in a classification that was based on average on 5.5 features rather than more than 70.000 features.
The performance of the SVM was evaluated using a 10-fold cross-validation. First, the data set was randomly split into 10 folds. $9 / 10$ folds were used to train the classifier, with a nested feature selection loop that used another 10-fold cross-validation within the training data to find the most predictive features. Then, the remaining 1/10, which were not included in the training and therefore also not in the feature selection, were classified, resulting in a certain classification accuracy. This procedure was then repeated, cycling through all folds, yielding a mean accuracy over the 10 folds. Because our groups were unbalanced regarding participant numbers, we randomly picked 84 data sets from the cohort of chronic pain patients for the classification procedure, repeating this 1000 times. To conclude whether this result truly exceeds chance level, we repeated the whole procedure with the same data, but the labels of chronic pain patients and healthy controls were randomly shuffled. ${ }^{12}$ Thus, we conducted 2000 analyses: 1000 with 84 randomly picked patients and the 84 healthy controls, and 1000 with the same data but randomly shuffled labels between patients and controls. The 2 resulting distributions of 1000 cross-validation accuracies each were then statistically compared using a nonparametric permutation test. ${ }^{46}$ The sensitivity is defined as the rate of true positives, ie, correctly classified patients, divided by the number of total patient classifications. The specificity is defined as the rate of true negatives, ie, correctly classified healthy controls, divided by the number of total healthy classifications.

Apart from the overall performance of the SVM, we also investigated which features contained the highest predictive value, ie, which features were most consistently picked by the SVMs by looking at the number of times a certain feature was included in the SVM by automatic feature selection.

\subsection{Statistical analysis}

Statistical analysis was performed using FieldTrip ${ }^{51}$ and customwritten Matlab scripts. The significance level for all statistical tests was set to 0.05 two-tailed. The underlying statistical test for all permutation tests was an unpaired T-test. We used clusterbased permutation tests with a cluster threshold of 0.05 to compare patients with healthy controls for all local analyses and the global power spectrum analysis. ${ }^{46}$ We report maximal and minimal T-values (t_max/min) for all cluster-based permutation tests that did not find any clusters. The other global measures were compared using nonparametric permutation tests, permuting between the patient group and healthy control group. ${ }^{46}$ To account for multiple comparisons within a specific measure, we corrected all $P$-values of tests that were performed on all 4 frequency bands using the Holm-Bonferroni method. ${ }^{33}$ To test whether the accuracy of the SVMs was above random chance level, we computed a nonparametric permutation test on the accuracy distribution of the SVM trained on the real data against the accuracy distribution of the SVM trained on the randomly labeled data. Here, a nonparametric permutation test was used to decrease the variety of different statistical tests. Pearson's $r$ was calculated to find correlations between brain measures and clinical parameters and tested for statistical significance against the null hypothesis of no correlation. Resulting $P$-values were again corrected for multiple comparisons across the 4 frequency bands using the Holm-Bonferroni method, if applicable.

\subsection{Data availability}

All scripts and data are available on request. 


\section{Results}

\subsection{Global measures of brain activity}

We first investigated whether chronic pain was associated with global changes of oscillatory brain activity. We determined the peak frequency of EEG activity in chronic pain patients and healthy controls by averaging the power spectra across all epochs and electrodes and determining the maximal power in the frequency range of 6 to $14 \mathrm{~Hz}$. Peak frequency was $9.8 \pm 1.2 \mathrm{~Hz}$ (mean \pm SD) in chronic pain patients and 10.0 $\pm 1.4 \mathrm{~Hz}$ in healthy controls and did not differ significantly between groups (nonparametric permutation test, $P=0.20$, Fig. 2A). Other common approaches to determine the peak frequency, by computing the center of gravity, analyzing longer time windows (5 seconds) for increased spectral resolution, or computing the peak frequency on individual epochs and then averaging, did not show a difference between groups either (nonparametric permutation tests, $P \geq 0.14$ )

Next, we examined whether chronic pain was associated with global changes of oscillatory brain activity at any frequency between 1 and $100 \mathrm{~Hz}$. To this end, we compared the global power spectrum of EEG activity averaged across all electrodes between chronic pain patients and healthy controls. The results did not reveal any significant difference between the 2 groups at any frequency (cluster-based permutation statistics clustered across frequencies, t_max $/ \min =1.7 /-1.5$; Fig. 2B). When controlling for intersubject differences in overall power by calculating power relative to the total power across all electrodes and frequencies for each participant, the results did not show a significant difference between patients and controls either (t_max/min $=1.4 /-1.7$ ).

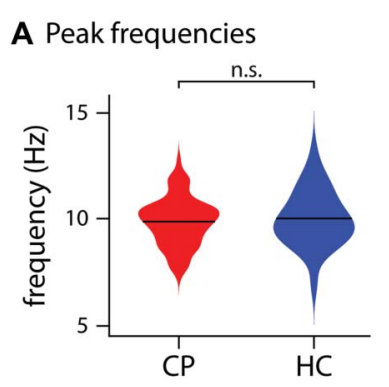

C Power topographies (CP - HC)

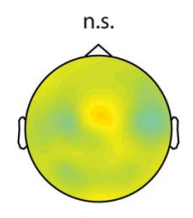

theta
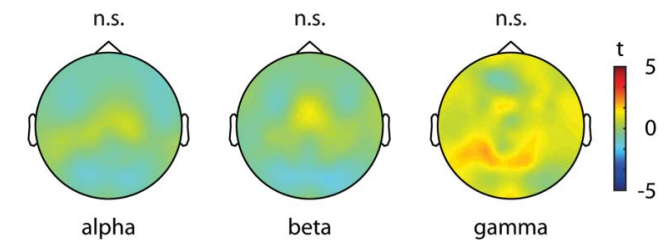

Figure 2. Global and local measures of brain activity. (A) Violin plot of the dominant peak frequencies computed on the average across all electrodes of chronic pain patients ( $\mathrm{CP}$, red) and healthy controls ( $\mathrm{HC}$, blue). A nonparametric permutation test showed no significant difference $(P=0.20)$ between the 2 groups. (B) Global power spectra of CP (red) and HC (blue), averaged across all electrodes and shown for the frequencies 1 to $100 \mathrm{~Hz}$, with a bandstop filter at 45 to $55 \mathrm{~Hz}$ to remove line noise. A cluster-based permutation test clustered across frequencies did not show any significant differences (t_max/min $=1.7 /-1.5)$. (C) Scalp topographies of power differences between $\mathrm{CP}$ and $\mathrm{HC}$ at theta, alpha, beta, and gamma frequencies, averaged across frequencies in each band. The colormap shows the $t$-values of a cluster-based permutation test. No significant clusters were found in any frequency band (thetat_max $/ \mathrm{min}=1.5 /-0.6$, alphat_max $/ \mathrm{min}=$ $0.62 /-1.2$, beta t_max $/ \min =1.0 /-1.4$, gamma t_max $/ \mathrm{min}=2.1 /-0.7$ ).
Thus, we did not observe global changes of the peak frequency or the power spectrum of oscillatory brain activity in chronic pain patients.

\subsection{Local measures of brain activity}

We further assessed whether chronic pain was associated with local changes of oscillatory brain activity. We therefore calculated topographical maps of brain activity for theta $(4-8 \mathrm{~Hz})$, alpha (8-13 $\mathrm{Hz})$, beta $(14-30 \mathrm{~Hz})$, and gamma $(60-100 \mathrm{~Hz})$ frequency bands. Group comparisons of the topographical maps did not show significant differences between patients and controls in any frequency band (cluster-based permutation statistics clustered across electrodes, t_max $/ \mathrm{min}=2.0 /-1.4$, Fig. $2 \mathrm{C}$ ). When controlling for intersubject differences in overall power by calculating relative power, the results did not show a significant difference between patients and controls either (t_max/min = 2.5/-3.2).

Thus, our findings did not show local changes of oscillatory brain activity in chronic pain patients in any frequency band.

\subsection{Local measures of functional connectivity}

Next, we explored whether chronic pain was associated with changes of functional connectivity, which is a measure of neural communication. To reduce potential confounds by field spread and volume conduction effects, ${ }^{60}$ we performed all connectivity analyses in source space using 2020 voxels with a size of $1 \times 1 \times$ $1 \mathrm{~cm}^{3}$. We first investigated whether chronic pain was associated with local changes of functional connectivity in any brain region or any frequency band. To this end, we calculated the connectivity strength for each voxel and frequency band. Connectivity strength was defined as the average connectivity of a specific voxel to all other voxels of the brain, which yields one connectivity strength value for each voxel. This allows for visualizing connectivity strength in a topographical map and applying the same statistical approaches used for the analysis of local brain activity. Analysis of phase-based connectivity (Fig. 3A) showed that chronic pain patients' connectivity strength in the theta band was significantly increased (cluster-based permutation test, $P$ [corrected/uncorrected] $=0.045 / 0.011$, Cohen's $d=0.40$ ) in comparison with the control group. The strongest contrast was found in the supplementary motor area $(\mathrm{MNI}=[-10,10,70])$. Moreover, we also found that patients showed a significantly increased connectivity strength in the gamma band (clusterbased permutation test, $P$ [corrected/uncorrected] $=0.0072$ / 0.0018 , Cohen's $d=0.59$ ), which was maximal in the anterior prefrontal cortex $(\mathrm{MNI}=[-40,40,30])$. Because in both frequency bands only a single extended cluster of increased connectivity was found, the increase likely reflects frontal connectivity within the clusters rather than connectivity to targets outside of the clusters. No significant clusters were found in the alpha and beta bands (alpha: $P$ _min [corrected/uncorrected] $=$ 1/0.61, t_max $=2.8$; beta: $P$ _min [corrected/uncorrected] $=$ $0.71 / 0.18$, t_max $=3.5$ ). Analysis of amplitude-based connectivity did not show any significant differences in connectivity strength between chronic pain patients and healthy controls in any brain region or any frequency band. (Fig. 3B, t_max/min = $0.4 /-1.2)$.

To further assess connectivity patterns of chronic pain patients, we performed graph theory-based network analysis. We first examined the local properties of brain networks in chronic pain patients. A basic property of a node is the number of its connections to other nodes, which is termed the degree. 
A Phase-based (PLV) connectivity (CP - HC)
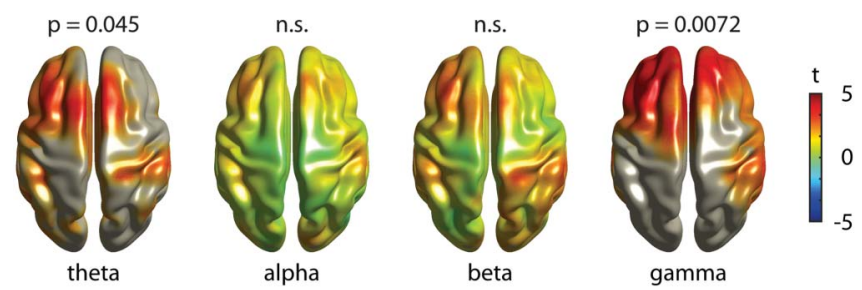

B Amplitude-based (AEC) connectivity (CP - HC)
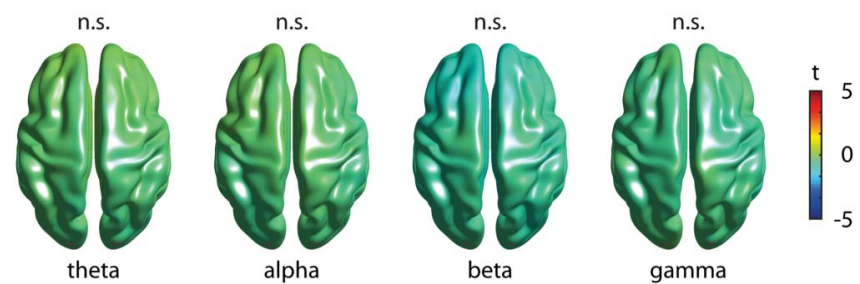

Figure 3. Local measures of functional connectivity. Brain topographies of the comparison of connectivity strength between chronic pain patients (CP) and healthy controls $(\mathrm{HC})$ in the theta, alpha, beta, and gamma band frequencies, averaged across frequencies in each band, are shown. Connectivity strength was calculated as the average connectivity of one voxel to all other voxels of the brain. The colormaps show the $t$-values. Significant results are masked, ie, all voxels but the ones belonging to a significant cluster are grayed out. When no significant clusters are found, nothing is grayed out to show potential trends. (A) Phase-based connectivity (phase locking value, PLV). A significant increase of chronic pain patients' connectivity strength was observed in the theta band $\left(P\right.$ [corrected/uncorrected] $=0.045 / 0.011, t \_\max =3.8$, Cohen's $\left.d=0.40\right)$ and the gamma band $(P$ [corrected/uncorrected] $=0.0072 / 0.0018$, t_max $=$ 5.1, Cohen's $d=0.59$ ). (B) Amplitude-based connectivity (orthogonalized amplitude envelope correlation, AEC). No significant differences were found in any frequency band (theta t_max $/ \mathrm{min}=0.4 /-0.6$, alpha t_max $/ \mathrm{min}=$ $0.1 /-0.7$, beta t_max $/ \min =-0.3 /-1.2$, gamma t_max $/ \min =0.0 /-1.1$ ).

Conceptually, the degree is closely related to the connectivity strength analyzed in the previous step, the essential difference being that the edges are thresholded, whereas the connectivity strength considers all connections. We computed the degree of every voxel and compared it between patients and controls. No difference in degree was found in any frequency band. This applied similarly to phase-based and amplitude-based measures of connectivity (PLV: P_min [corrected/uncorrected] $=0.51 / 0.13$, t_max $=4.2 ;$ AEC: $P \_\min [$ corrected/uncorrected] $=1 / 0.56$, t_max = 3.0). In addition, we computed the weighted degree, ie, a thresholded but not binarized connectivity matrix. Clusterbased permutation tests of the weighted degree showed no significant differences between patients and controls in any frequency band either (PLV: P_min [corrected/uncorrected] $=$ 0.24/0.061, t_max $=4.3$; AEC: $P \_\min [$ corrected/uncorrected] $=$ $1 / 0.31$, t_max $=3.2$ ). This lack of a difference in (weighted) degree indicates that the difference in connectivity strength is not confined to the strongest connections but instead applies to connections of all strengths.

Another well-established measure that characterizes nodes is the CC. This measure assesses the number of connections of neighboring nodes, ie, it measures the local integration of a node served by short-range connectivity. Comparing the CCs of all nodes between patients and controls did not show any significant differences at any frequency band, neither for phase-based nor amplitude-based connectivity (PLV: P_min [corrected/ uncorrected] $=0.12 / 0.030$, t_max $=3.3$, AEC: $P$ _min [corrected/uncorrected] $=1 / 0.28$, t_max $=3.8$ ).

Taken together, the analysis of local measures of functional connectivity showed increases of phase-based connectivity in frontal and prefrontal cortices at theta and gamma frequencies in chronic pain patients. The increase in the theta band was of small effect size (Cohen's $d=0.40$ ), whereas the increase in the gamma band was of medium effect size (Cohen's $d=0.59$ ).

\subsection{Global measures of functional connectivity}

We next investigated whether chronic pain was associated with changes of global measures of functional connectivity and therefore computed graph measures that characterize different and complementary global properties of brain networks. Figure 4 summarizes the results of the global graph measures. First, we calculated the $\mathrm{gCC}$, which is commonly interpreted as a measure of functional segregation in a network. We found no differences in gCC between chronic pain patients and healthy controls (Table 3; $P$ _min [corrected/uncorrected] $=0.088 / 0.022$ ). Second, we assessed the global efficiency, which provides an account of the ease of long-distance communication and is commonly interpreted as a measure of functional integration in a network. After accounting for multiple comparisons, we found evidence for a decrease of global efficiency in patients in the gamma frequency band when investigating phase-based connectivity (Table 3; $P$ [corrected/uncorrected] $=0.013 / 0.0032$ ). The effect size of this difference was small (Cohen's $d=0.44$ ). Third, we computed the small-worldness, which is associated with communication efficiency within a network. We detected no changes in small-worldness between the 2 groups (Table 3; $P \_$min [corrected/uncorrected] $\left.=0.26 / 0.064\right)$. Fourth, we analyzed the hub disruption index, which has been shown to differ between chronic pain patients and controls in previous functional magnetic resonance imaging studies. ${ }^{44,45}$ It shows potential shifts of connections that manifest on a global scale. Our results did not show a difference of the hub disruption index in any frequency band when comparing chronic pain patients with healthy controls (Table 3; $P$ _min [corrected/uncorrected] $=$ 1/0.32).

In summary, global graph measures of functional connectivity showed a decrease of global efficiency at gamma frequencies in chronic pain patients. This decrease was of small effect size (Cohen's $d=0.44$ ).

\subsection{Additional functional connectivity analyses}

First, as muscle artifacts may cause spurious synchrony, we have analyzed connectivity between EMG signals and EEG signals. Electromyography signals were recorded from the right neck and masseter muscles and band-pass filtered at theta $(4-8 \mathrm{~Hz})$ and gamma $(60-100 \mathrm{~Hz})$ frequencies. Electroencephalography signals were band-pass filtered source signals from peak voxels of clusters where statistically significant group differences in EEG connectivity were found at theta (MNI coordinates $\left.=\left[\begin{array}{lll}-10 & 10 & 70\end{array}\right]\right)$ and gamma ( $\mathrm{MNI}$ coordinates $=\left[\begin{array}{lll}-40 & 40 & 30\end{array}\right]$ ) frequencies. Statistical comparisons of PLV-based EMG-EEG connectivity did not show any significant differences between chronic pain patients and healthy controls. In particular, nonparametric permutation tests comparing EMG-EEG connectivity bandpass filtered in the theta band $(4-8 \mathrm{~Hz})$ between groups yielded $P$-values of 0.3 (neck muscles) and 0.08 (masseter). Group comparisons of EMG-EEG connectivity band-pass filtered in the gamma band $(60-100 \mathrm{~Hz}$ ) yielded $P$-values of 0.6 (neck muscles) and 0.7 (masseter). These findings argue against a contamination of the observed connectivity differences between chronic pain patients and healthy controls by muscle artifacts. 
A Phase-based (PLV) connectivity

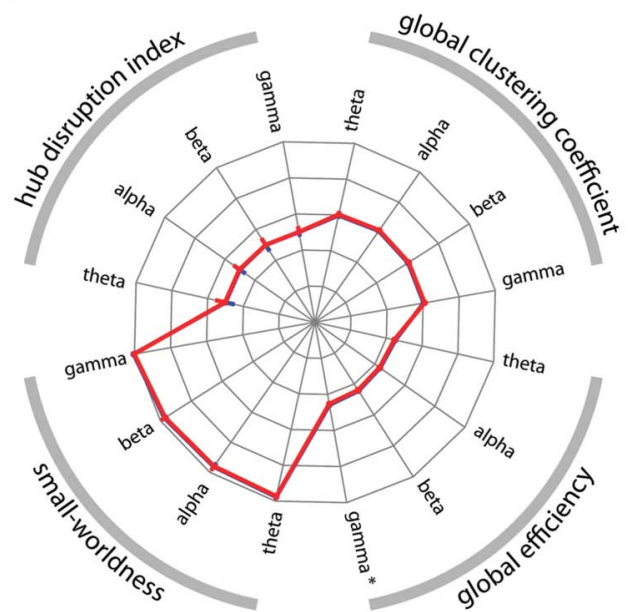

B Amplitude-based (AEC) connectivity

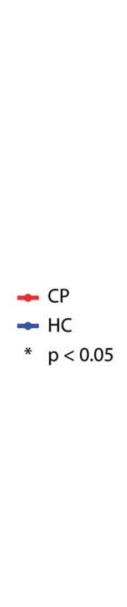

B Amplitude-based (AEC) connectivity

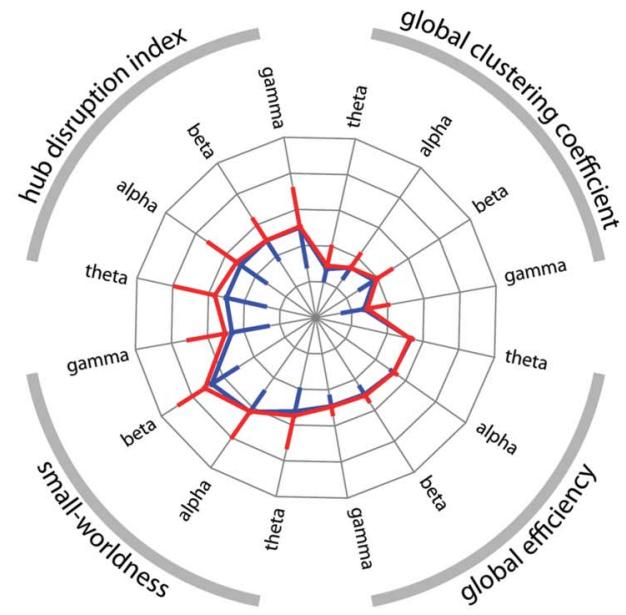

Figure 4. Global graph theoretical measures of functional connectivity. The radar plots show 4 global graph measures in 4 frequency bands based on (A) phase-based and (B) amplitude-based connectivity measures. The clockwise arrangement follows the following pattern: theta, alpha, beta, and gamma repeated for the 4 graph measures: global clustering coefficient, global efficiency, small-worldness, and absolute values of the hub disruption index. The red lines show the chronic pain patients' (CP) values, whereas the blue lines represent the healthy controls' (HC) values. Error bars show the $\mathrm{SD}$. For visualization purposes, the symmetric error bars are only drawn in a single radial direction. Axes run from the center $(=0)$ to the outside $(=1)$. For visualization purposes, the small-worldness and hub disruption index were scaled with a factor of 0.2. (A) Phase-based connectivity (phase locking value, PLV). The global efficiency in the gamma band was significantly decreased in chronic pain patients (nonparametric permutation test, $P$ [corrected/uncorrected $]=0.013 / 0.0032$, Cohen's $d=0.44)$. No other measure revealed a significant difference when compared between groups, see Table 3 for details. (B) Amplitude-based connectivity (orthogonalized amplitude envelope correlation, AEC). No significant difference between groups was observed, Table 3 for statistical details.

Second, we tested whether changes of functional connectivity in chronic pain patients can be detected using another common phase-based connectivity measure (dwPLI). ${ }^{66,78}$ The dwPLI differs from the PLV by capturing nonzero phase lag connectivity only. The dwPLI is therefore not susceptible to volume conduction, which can yield artificial connectivity effects at the cost of reduced sensitivity because real synchrony at zero phase lag is also discarded. The results of the cluster-based permutation tests did not reveal any local difference in functional connectivity between patients and controls ( $P$ _min [corrected/ uncorrected] $=0.48 / 0.12$, t_ $\min =-3.0$ ). This indicates that zero phase lag connectivity plays an important role in the increased frontal connectivity of patients. Concerning global graph measures (Table 4), the hub disruption index was significantly lower in chronic pain patients in the gamma band $(P$ [corrected/ uncorrected] $<0.001 /<0.001$, Cohen's $d=0.63$ ). This difference together with a lack of a difference in the corresponding

\section{Table 3}

Comparisons of global graph measures of functional connectivity between chronic pain patients and healthy controls.

\begin{tabular}{clllllllll}
\hline & PLV & & & & & AEC \\
\cline { 2 - 3 } \cline { 7 - 9 } & Theta & Alpha & Beta & Gamma & Theta & Alpha & Beta & Gamma \\
\hline gCC & 0.084 & 0.040 & 0.092 & 0.022 & 0.22 & 0.92 & 0.29 & 0.38 \\
\hline gEff & 0.052 & 0.13 & 0.084 & 0.0032 & 0.17 & 0.78 & 0.30 & 0.74 \\
\hline$S$ & 0.17 & 0.24 & 0.24 & 0.064 & 0.30 & 0.92 & 0.094 & 0.24 \\
\hline$k_{d}$ & 0.77 & 0.64 & 0.94 & 0.88 & 0.066 & 0.32 & 0.99 & 0.88 \\
\hline
\end{tabular}

Uncorrected $P$-values of nonparametric permutation tests comparing global graph measures between groups. After correction for multiple comparisons using the Holm-Bonferroni method across the 4 frequency bands to take cross-spectral dependencies into account, only the PLV-based global efficiency in the gamma band was significantly lower in patients. ( $P$ [corrected $]=0.013$, Cohen's $d=0.44$ ).

AEC, amplitude envelope correlation; gCC, global clustering coefficient; gEff, global efficiency; $k_{d}$, hub disruption index; PLV, phase locking value; S, small-worldness.
PLV-based analysis indicates that the difference mainly applies to nonzero phase lag connectivity.

Third, we performed control network analyses by calculating all graph measures with different edge densities using all 3 connectivity measures. This was done to examine the robustness of our results, which were based on a threshold of $10 \%$ strongest connections. No significant group differences in local graph measures were found for any of the connectivity measures. Regarding global measures, the global efficiency in the gamma band calculated with the PLV (5\% edge density, $P$ [corrected] $=$ 0.011 , Cohen's $d=0.44 ; 20 \%$ edge density, $P$ [corrected] $=$ 0.0080 , Cohen's $d=0.44$ ) and the hub disruption index in the gamma band calculated with the dwPLI (5\% edge density, $P$ [corrected] $<0.001$, Cohen's $d=0.61 ; 20 \%$ edge density, $P$ [corrected] $<0.001$, Cohen's $d=0.61$ ) were both significantly lower in chronic pain patients irrespective of edge density and therefore showed a consistent and robust effect.

\section{Table 4}

Comparisons of global graph measures between chronic pain patients and healthy controls-debiased weighted phase lag index (dwPLI).

\begin{tabular}{lllll}
\hline & dwPLI & & & \\
\cline { 2 - 5 } & Theta & Alpha & Beta & Gamma \\
\hline $\mathrm{gCC}$ & 0.088 & 0.45 & 0.017 & 0.47 \\
\hline $\mathrm{gEff}$ & 0.29 & 0.051 & 0.20 & 0.12 \\
\hline $\mathrm{S}$ & 0.085 & 0.30 & 0.090 & 0.29 \\
\hline $\mathrm{k}_{d}$ & 0.048 & 0.20 & 0.43 & $<0.001$ \\
\hline
\end{tabular}

Uncorrected $P$-values of nonparametric permutation tests comparing global graph measures between groups. After correction for multiple comparisons using the Holm-Bonferroni method across the 4 frequency bands to take cross-spectral dependencies into account, only the dwPLI-based hub disruption index $\left(\mathrm{k}_{\mathrm{d}}\right)$ in the gamma band was significantly lower in patients $(P$ [corrected] $=0.00$, Cohen's $d=0.63$ )

gCC, global clustering coefficient; gEff, global efficiency; $k_{d}$, hub disruption index; S, small-worldness. 
Finally, we tested whether depression plays a critical role in explaining differences between patients and controls. We therefore aimed to replicate the significant findings after excluding patients with a clinically relevant depression (BDI-II score $\geq 18, n$ $=36$ ). This reanalysis did not qualitatively change any of the previously significant results. Frontal connectivity was increased for chronic pain patients without depression in the theta $(P$ [corrected] $=0.0080$, Cohen's $d=0.65)$ and gamma $(P$ [corrected] $=0.0048$, Cohen's $d=0.68$ ) bands. Similarly, PLVbased global efficiency ( $P$ [corrected] $=0.014$, Cohen's $d=0.42$ ) and the dwPLI-based hub disruption index ( $P$ [corrected] $<0.001, d=0.75$ ) were decreased at gamma frequencies in chronic pain patients without depression.

In summary, the PLV global efficiency and the dwPLI hub disruption index in the gamma band were consistently changed in chronic pain patients even when excluding patients with depression. Both measures were decreased in chronic pain patients, the PLV global efficiency showing a small effect size and the dwPLI hub disruption index showing a medium effect size.

\subsection{Relationship between brain activity/functional connectivity and clinical parameters}

We further investigated the relationships of brain-based activity and connectivity measures with clinical parameters. To reduce the number of statistical tests, we restrained our analyses to selected measures of brain activity and brain connectivity, which were associated with clinical parameters of chronic pain patients in previous studies. ${ }^{10,17,21,28,39,45,58,59,67,75}$ We thus computed correlations between the global peak frequency, mean global power in the 4 frequency bands, the hub disruption index, and the following major clinical parameters: current pain intensity, average pain intensity in the past 4 weeks, pain duration, pain disability, mental and physical quality of life, depression, and medication as quantified by the medication quantification scale. In addition, we correlated the significant clusters in the theta and gamma PLV connectivity, the PLV global efficiency in the gamma band, and the dwPLI hub disruption index with the same clinical parameters. The results showed no significant correlations (Fig. 5). Thus, we did not observe any relations of measures of brain activity and functional connectivity with clinical parameters including medication. This suggests that increases of frontal connectivity and global network changes in chronic pain patients do not scale with disease characteristics but rather characterize the state of chronic pain per se.

\subsection{Machine learning approach}

Finally, we performed a multivariate machine learning approach. This approach extends the previous univariate approaches by taking patterns of brain activity and connectivity into account rather than single pieces of information in isolation. Moreover, it complements the previous descriptive group analyses by adding a predictive, singlesubject analysis. We used an SVM classifier to test whether patterns of brain activity and/or connectivity can distinguish between chronic pain patients and healthy controls. We trained a linear SVM on all aforementioned measures of brain activity and functional connectivity, using an automated sequential feature selection algorithm. The

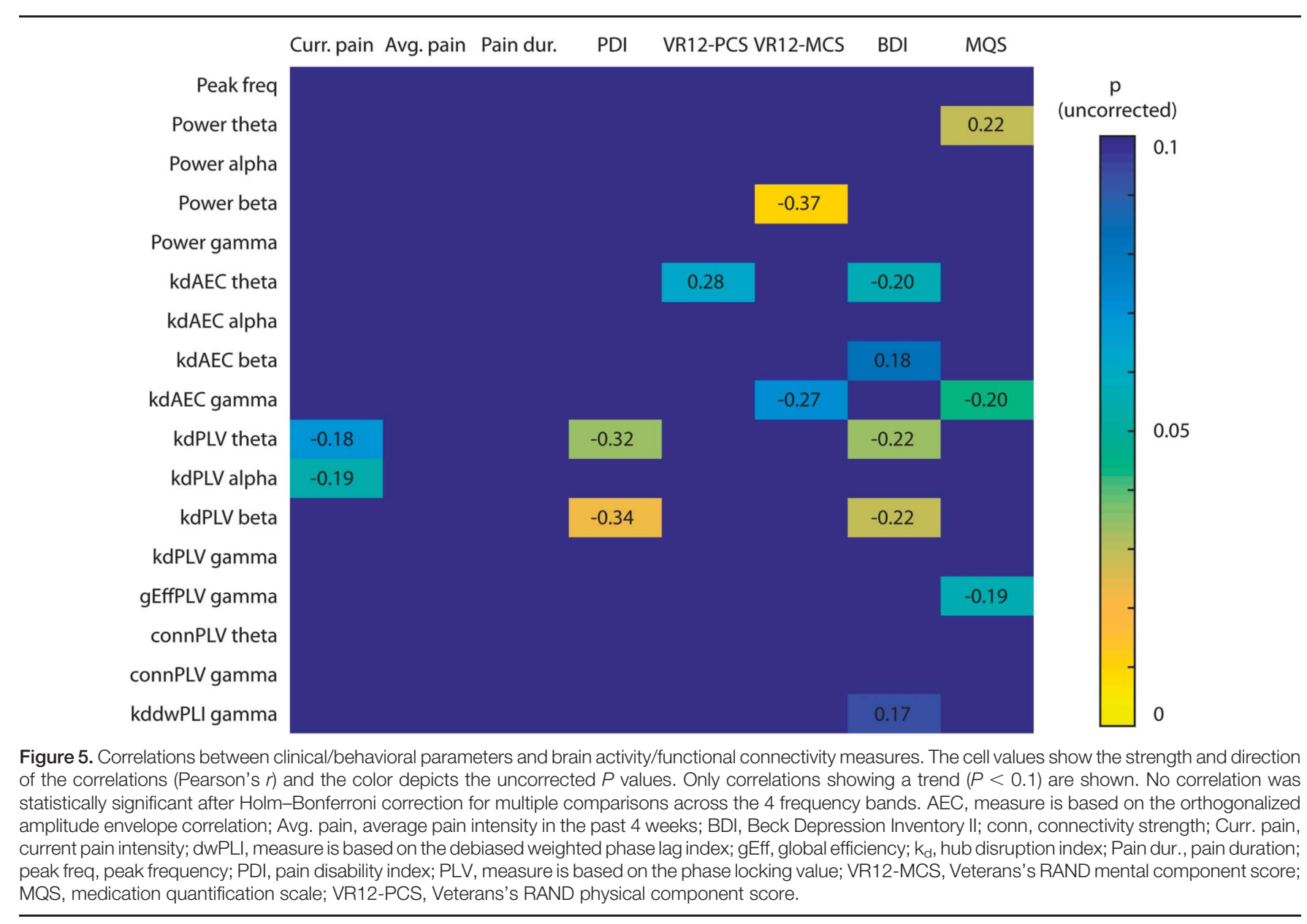


performance of the SVM was evaluated using a 10-fold crossvalidation. The resulting mean accuracy was $57 \% \pm 4 \%$ with a sensitivity of $60 \% \pm 5 \%$ and a specificity of $57 \% \pm 5 \%$. To test whether this result exceeds chance level, we repeated the whole procedure with the same data but randomly shuffled labels of chronic pain patients and healthy controls. This resulted in a permutation distribution with 50\% $\pm 5 \%$ accuracy. A nonparametric permutation test of the 2 accuracy distributions (Fig. 6A) confirmed that the real model was significantly more accurate than random guessing $(P<$ 0.001). Finally, we were interested to know which features of brain activity and/or connectivity were most relevant for the classification. The automatic feature selection on average picked 5.5 features for the SVMs. We therefore show the 5 most frequently picked features in Figure 6B. These features were chosen with a rate of $10 \%$ to $15 \%$ each and all 5 are measures of phase-based connectivity (PLV or dwPLI). Thus, in more than 50\% of classifications, phase-based connectivity in frontal brain areas was chosen by the SVMs. The most relevant features were phase-based connectivity measures in frontal brain areas at gamma ( $\mathrm{MNI}:-40,30,40$ and $-30,50,10)$ and theta (MNI: -20, 50, 40) frequencies.

Thus, a multivariate machine learning approach could statistically significantly distinguish between chronic pain patients and healthy controls based on EEG measures of brain activity and connectivity. In particular, frontal phase-based connectivity at theta and gamma frequencies provided important information for the classification.

\section{Discussion}

In this study, we systematically exploited the potential of EEG to determine abnormalities of brain function in chronic pain. Defining

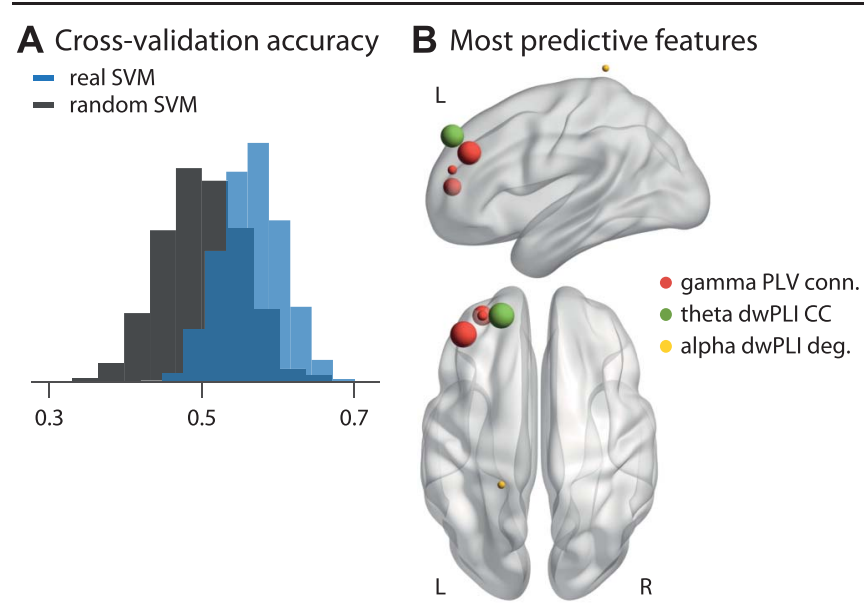

Figure 6. Multivariate machine learning approach to classify chronic pain patients and healthy controls. (A) Distribution of mean accuracies resulting from a 10-fold cross-validation. The blue histogram shows the results trained on the actual data including all features of brain activity and connectivity. The gray histogram shows a support vector machine (SVM) trained on data with randomly permuted labels. The SVM trained on the real data shows an accuracy of $57 \pm 4 \%$, significantly higher than the accuracy of the SVM trained on randomly permuted data, $50 \pm 5 \%(P<0.001)$. (B) The 5 most predictive features, ie, those selected most consistently by the SVMs. Specific measures are color-coded, and the size of the spheres represents how often a specific feature was selected. The most frequently selected features were phase locking value (PLV)-based connectivity of the prefrontal cortex (MNI: $-40,30$, 40 and $-30,50,10$ ) in the gamma band, which were selected in $15 \%$ and $12 \%$ of SVMs, respectively, and debiased weighted phase lag index (dwPLI) based clustering coefficient of the prefrontal cortex (MNI: $-20,50,40)$ in the theta band, which was selected in $15 \%$ of SVMs. All other features were selected with a frequency of less than 10\%. MNI, Montreal Neurological Institute. such abnormalities promises to advance the understanding of the neural basis of chronic pain. Moreover, they might serve as a brain-based marker and novel treatment target of chronic pain. To this end, we analyzed resting-state EEG recordings of a large cohort of patients suffering from chronic pain and compared them with those of age- and sex-matched healthy controls. The analyses ranged from simple global measures of brain activity to sophisticated connectivity and network analyses in source space. All analyses were data-driven and each analysis was rigorously corrected for multiple comparisons. To the very best of our knowledge, this approach represents the most extensive analysis of EEG data from one of the largest cohorts of chronic pain patients so far. The results show that global measures of brain activity and brain connectivity as measured by EEG did not differ between chronic pain patients and a healthy control group. However, our approach revealed a stronger phasebased connectivity at theta and gamma frequencies in the prefrontal cortex of chronic pain patients. Furthermore, we observed a global reorganization of brain networks in the gamma frequency band. Based on patterns of brain activity and connectivity, a multivariate machine learning approach could classify chronic pain patients and healthy controls with an accuracy of $57 \%$.

Previous resting-state EEG studies investigating alterations in chronic pain patients mainly reported an increase in theta power together with a slowing of the global peak frequency compared with healthy controls. ${ }^{7,58,76,79}$ These findings have been related to the TCD model of chronic pain. ${ }^{41,42}$ In this model, abnormal nociceptive input causes abnormal thalamic bursts at theta frequencies. These theta oscillations are transmitted to the cerebral cortex where they result in disinhibition of neighboring areas, abnormal oscillations at gamma frequencies, and eventually in ongoing pain. This model is highly appealing, but evidence is still sparse. The present completely data-driven approach in a large cohort of chronic pain patients neither shows increased theta power nor a shift of global peak frequency and therefore does not directly support the TCD model of chronic pain.

The univariate comparisons of brain activity/connectivity between groups and the multivariate machine learning approach congruently indicated increased functional connectivity of the prefrontal cortex in chronic pain patients. These findings are in accordance with $\mathrm{fMRl}^{3,31,72}$ and $\mathrm{EEG}^{47}$ studies as well as with recent reviews and theories, 2,55,62 which have shown that structural and functional alterations in the prefrontal cortex play an important role in chronic pain. A more precise localization of the connectivity increases in the prefrontal cortex is beyond the spatial resolution of EEG. Hence, it remains unclear how the present observations relate to the multitude of functions represented in the prefrontal cortex, which include motor, cognitive control, emotional, evaluative, and modulatory functions. ${ }^{16,37}$ However, a role of the prefrontal cortex in chronic pain points to an important function of emotional-evaluative, motivational, and decision-making circuits rather than sensory circuits in chronic pain. ${ }^{2,55}$

Our findings revealed that chronic pain is associated with frontal connectivity increases and a global disturbance of brain network organization at gamma frequencies. Mechanistically, gamma oscillations have been related to the activity of inhibitory parvalbumin-positive GABAergic interneurons. ${ }^{9}$ In an animal model of chronic pain, these interneurons have been implicated in the modulation of pyramidal cell firing in the prefrontal cortex and pain behavior. ${ }^{85}$ This link between GABAergic inhibition, gamma oscillations, prefrontal cortex activity, and pain behavior is in accordance with the present observations. Functionally, 
gamma oscillations likely represent a basic feature of neuronal signaling and communication, ${ }^{19,25,81}$ which seems to be particularly related to the local processing and feedforward communication of currently important stimuli. ${ }^{19,25,53}$ These concepts would be in line with an association of chronic pain with prefrontal gamma oscillations possibly signaling the emotional, motivational, and evaluative aspects of pain.

In addition, decreases of global efficiency and hub disruption index in the gamma band indicate that chronic pain is not only associated with local changes of functional connectivity but also a global reorganization of brain networks. This is in accordance with recent $\mathrm{fMRl}$ studies that have shown global changes of functional connectivity at infraslow frequencies below $0.1 \mathrm{~Hz} .{ }^{44,45}$ The present findings complement these studies by showing that global changes of functional connectivity also occur in the gamma band at much higher frequencies between 60 and $100 \mathrm{~Hz}$.

The present machine learning approach shows that applying an SVM classifier to resting-state EEG data allows to distinguish between chronic pain patients and healthy controls with $57 \%$ accuracy. A recent study that pursued a closely related EEG approach showed accuracies of $>90 \%$ for the classification of chronic pain patients vs healthy controls, ${ }^{76}$ which were not achieved in this study. The reasons for this disparity remain unclear because the available information on the previous approach does not allow for precise replication. Although the accuracy of the present study is far from being sufficient for practical purposes, this result has important implications. First, it indicates that prefrontal synchrony at theta and gamma frequencies might play a role in the pathophysiology of chronic pain. Second, the present approach might represent a step further towards a much sought-after brain-based marker of pain. ${ }^{15,71} \mathrm{fMRI}$ recordings have already shown that, in principle, it is possible to establish such a marker. ${ }^{44,80}$ The present approach complements these $\mathrm{fMRI}$ approaches by using EEG recordings. Third, abnormal patterns of EEG activity in chronic pain might represent potential targets for novel therapeutic strategies such as non-invasive brain stimulation techniques ${ }^{54}$ and/or neurofeedback approaches. ${ }^{63}$ In particular, the emerging transcranial alternating current stimulation technique ${ }^{54}$ allows for the frequency-specific modulation of neuronal oscillations and synchrony and might, thus, represent a promising approach to modulate pain.

Several limitations of this study need to be pointed out. First, abnormal oscillations and synchrony are observed in many neurological diseases including depression ${ }^{69,70}$ and the specificity of the present results for chronic pain remains unclear. However, changes of prefrontal theta and gamma synchrony were similarly found when patients with depression were excluded. Moreover, the causal relationship between the observed changes of brain function and chronic pain remains unclear. However, a potential lack of specificity and/or causality does not necessarily limit the clinical usefulness and validity of a brain-based marker of chronic pain. ${ }^{15}$ Second, field spread and/or muscle artifacts can cause spurious synchrony of EEG signals. A rigorous artifact correction procedure and analysis in source space are best practice to reduce these effects. Moreover, the present analyses are based on comparisons between groups, and systematic differences of volume conduction between groups are unlikely. Furthermore, a lack of group differences in amplitude of oscillatory of brain activity argues against volume conduction effects on the observed group differences in connectivity. In addition, a lack of connectivity between EMG and EEG signals argues against a contamination of the observed differences in EEG connectivity by EMG signals.
Nevertheless, muscle artifacts and volume conduction remain an inherent and delicate confound of EEG signals. Third, drug effects cannot be ultimately ruled out. We excluded patients taking benzodiazepines, which have known effects on EEG signals. ${ }^{4}$ However, in our representative cohort of chronic pain patients, most patients took nonopioid analgesics, opioids, and/or antidepressants. To control for drug effects, we quantified medication and found no significant correlations between medication and the observed EEG effects. Therefore, it is unlikely but not impossible that our effects are solely driven by drug effects. Fourth, for each measure of brain activity and connectivity, we have corrected for multiple comparisons across frequency and/or space. However, we have not corrected for multiple comparisons across the different measures of brain activity and brain connectivity. This has been done to decrease the risk of false-negative findings. However, it increases the risk of false-positive findings so that the current findings need replication and reproduction.

In conclusion, our data-driven, systematic, and extensive analysis of EEG data from a large cohort of chronic pain patients shows that local and global measures of brain activity did not differ between chronic pain patients and a healthy control group. These negative findings might help to clarify inconsistencies in previous studies and guide future research. Moreover, our study reveals increased prefrontal synchrony together with global network reorganization at gamma frequencies in chronic pain, which allows for differentiating chronic pain patients from healthy controls. These findings advance the understanding of the brain mechanisms of chronic pain. Beyond, the present observations might represent a step further towards a safe, low-cost, broadly available, and potentially mobile brain-based marker of pain. However, substantial challenges concerning the possibility of false-positive findings and the accuracy, specificity, and validity of such a marker remain to be overcome. Finally, the findings might open new therapeutic perspectives by revealing a potential target for novel noninvasive brain stimulation and neurofeedback strategies.

\section{Conflict of interest statement}

The authors have no conflicts of interest to declare.

\section{Acknowledgments}

The authors thank all patients and healthy participants for their participation in the study. Special thanks to Christine Berger and Vesna Dolanjski for support with patients and Marlene Försterling for help with data acquisition. The authors thank Ulrike Bingel and Valentin Riedl for helpful comments on the manuscript. The study was supported by the Deutsche Forschungsgemeinschaft (PL 321/10-1, PL 321/11-1, PL 321/10-2, PL 321/11-2), the Bavarian State Ministry of Education, Science and the Arts, and the Wellcome Trust (098433).

\section{Article history:}

Received 6 March 2019

Received in revised form 11 June 2019

Accepted 17 July 2019

Available online 22 July 2019

\section{References}

[1] Achard S, Delon-Martin C, Vértes PE, Renard F, Schenck M, Schneider F, Heinrich C, Kremer S, Bullmore ET. Hubs of brain functional networks are 
radically reorganized in comatose patients. Proc Natl Acad Sci USA 2012; 109:20608-13.

[2] Baliki MN, Apkarian AV. Nociception, pain, negative moods, and behavior selection. Neuron 2015;87:474-91.

[3] Baliki MN, Petre B, Torbey S, Herrmann KM, Huang L, Schnitzer TJ, Fields HL, Apkarian AV. Corticostriatal functional connectivity predicts transition to chronic back pain. Nat Neurosci 2012;15:1117-9.

[4] Bauer G, Bauer R. EEG, drug effects, and central nervous system poisoning. In: Schomer DL, da Silva FHL, editors. Niedermeyer's electroencephalography: basic principles, clinical applications, and related fields. Philadelphia: Oxford University Press, 2011. pp. 901-22.

[5] Bazanova OM, Vernon D. Interpreting EEG alpha activity. Neurosci Biobehav Rev 2014:44:94-110.

[6] Beck AT, Steer RA, Brown G. Manual for the beck depression inventory-II. San Antonio: Psychological Corporation, 1996.

[7] Boord P, Siddall PJ, Tran Y, Herbert D, Middleton J, Craig A. Electroencephalographic slowing and reduced reactivity in neuropathic pain following spinal cord injury. Spinal Cord 2008;46:118-23.

[8] Breivik H, Collett B, Ventafridda V, Cohen R, Gallacher D. Survey of chronic pain in Europe: prevalence, impact on daily life, and treatment. Eur J Pain 2006;10:287-333.

[9] Buzsáki G, Wang XJ. Mechanisms of gamma oscillations. Annu Rev Neurosci 2012;35:203-25.

[10] Choe MK, Lim M, Kim JS, Lee DS, Chung CK. Disrupted resting state network of fibromyalgia in theta frequency. Sci Rep 2018;8:2064.

[11] Chu CJ, Kramer MA, Pathmanathan J, Bianchi MT, Westover MB, Wizon L, Cash SS. Emergence of stable functional networks in long-term human electroencephalography. J Neurosci 2012;32:2703-13.

[12] Combrisson E, Jerbi K. Exceeding chance level by chance: the caveat of theoretical chance levels in brain signal classification and statistical assessment of decoding accuracy. J Neurosci Methods 2015;250: 126-36.

[13] Cortes C, Vapnik V. Support-vector networks. Machine learning 1995;20: 273-97.

[14] Davis JF. Manual of surface electromyography. WADC Technical Report (59-184). Montreal, 1959.

[15] Davis KD, Flor H, Greely HT, lannetti GD, Mackey S, Ploner M, Pustilnik A, Tracey I, Treede RD, Wager TD. Brain imaging tests for chronic pain: medical, legal and ethical issues and recommendations. Nat Rev Neurol 2017;13:624-38.

[16] de la Vega A, Chang LJ, Banich MT, Wager TD, Yarkoni T. Large-scale meta-analysis of human medial frontal cortex reveals tripartite functional organization. J Neurosci 2016;36:6553-62.

[17] de Vries M, Wilder-Smith OH, Jongsma ML, van den Broeke EN, Arns M, van Goor H, van Rijn CM. Altered resting state EEG in chronic pancreatitis patients: toward a marker for chronic pain. J Pain Res 2013;6:815-24.

[18] Dillmann U, Nilges P, Saile H, Gerbershagen HU. Assessing disability in chronic pain patients [in German]. Schmerz 1994;8:100-10.

[19] Donner TH, Siegel M. A framework for local cortical oscillation patterns. Trends Cogn Sci 2011;15:191-9.

[20] Engel AK, Gerloff C, Hilgetag CC, Nolte G. Intrinsic coupling modes: multiscale interactions in ongoing brain activity. Neuron 2013;80:867-86.

[21] Fallon N, Chiu Y, Nurmikko T, Stancak A. Altered theta oscillations in resting EEG of fibromyalgia syndrome patients. Eur J Pain 2018;22: 49-57.

[22] Faul F, Erdfelder E, Lang AG, Buchner A. G. *Power 3: a flexible statistical power analysis program for the social, behavioral, and biomedical sciences. Behav Res Methods 2007;39:175-91.

[23] Fornito A, Zalesky A, Bullmore ET. Fundamentals of brain network analysis. San Diego: Academic Press, 2016.

[24] Freynhagen R, Baron R, Gockel U, Tölle TR. painDETECT: a new screening questionnaire to identify neuropathic components in patients with back pain. Curr Med Res Opin 2006;22:1911-20.

[25] Fries P. Rhythms for cognition: communication through coherence. Neuron 2015;88:220-35.

[26] Furman AJ, Meeker TJ, Rietschel JC, Yoo S, Muthulingam J, Prokhorenko M, Keaser ML, Goodman RN, Mazaheri A, Seminowicz DA. Cerebral peak alpha frequency predicts individual differences in pain sensitivity. Neuroimage 2017;167:203-10.

[27] Global Burden of Disease Study C. Global, regional, and national incidence, prevalence, and years lived with disability for 328 diseases and injuries for 195 countries, 1990-2016: a systematic analysis for the Global Burden of Disease Study 2016. Lancet 2017:390:1211-59.

[28] Gonzalez-Roldan AM, Cifre I, Sitges C, Montoya P. Altered dynamic of EEG oscillations in fibromyalgia patients at rest. Pain Med 2016;17: 1058-68.

[29] Gross J, Baillet S, Barnes GR, Henson RN, Hillebrand A, Jensen O, Jerbi K, Litvak V, Maess B, Oostenveld R, Parkkonen L, Taylor JR, van
Wassenhove V, Wibral M, Schoffelen JM. Good practice for conducting and reporting MEG research. Neuroimage 2013;65:349-63.

[30] Harden RN, Weinland SR, Remble TA, Houle TT, Colio S, Steedman S, Kee WG. Medication Quantification Scale Version III: update in medication classes and revised detriment weights by survey of American Pain Society Physicians. J Pain 2005;6:364-71.

[31] Hashmi JA, Baliki MN, Huang L, Baria AT, Torbey S, Hermann KM, Schnitzer TJ, Apkarian AV. Shape shifting pain: chronification of back pain shifts brain representation from nociceptive to emotional circuits. Brain 2013;136:2751-68.

[32] Hipp JF, Hawellek DJ, Corbetta M, Siegel M, Engel AK. Large-scale cortical correlation structure of spontaneous oscillatory activity. Nat Neurosci 2012:15:884-90.

[33] Holm S. A simple sequentially rejective multiple test procedure. Scand J Statist 1979;6:65-70.

[34] Jung TP, Makeig S, Humphries C, Lee TW, McKeown MJ, Iragui V, Sejnowski TJ. Removing electroencephalographic artifacts by blind source separation. Psychophysiology 2000;37:163-78.

[35] Kennedy J, Roll JM, Schraudner T, Murphy S, McPherson S. Prevalence of persistent pain in the U.S. adult population: new data from the 2010 national health interview survey. J Pain 2014;15:979-84.

[36] Klimesch W, Schimke H, Pfurtscheller G. Alpha frequency, cognitive load and memory performance. Brain Topogr 1993;5:241-51.

[37] Kragel PA, Kano M, Van Oudenhove L, Ly HG, Dupont P, Rubio A, DelonMartin C, Bonaz BL, Manuck SB, Gianaros PJ, Ceko M, Reynolds Losin EA, Woo CW, Nichols TE, Wager TD. Generalizable representations of pain, cognitive control, and negative emotion in medial frontal cortex. Nat Neurosci 2018;21:283-9.

[38] Kuner R, Flor H. Structural plasticity and reorganisation in chronic pain. Nat Rev Neurosci 2017; 18:113.

[39] Kuo PC, Chen YT, Chen YS, Chen LF. Decoding the perception of endogenous pain from resting-state MEG. Neuroimage 2017;144:1-11.

[40] Lachaux JP, Rodriguez E, Martinerie J, Varela FJ. Measuring phase synchrony in brain signals. Hum Brain Mapp 1999;8:194-208.

[41] Llinás R, Urbano FJ, Leznik E, Ramírez RR, van Marle HJ. Rhythmic and dysrhythmic thalamocortical dynamics: GABA systems and the edge effect. Trends Neurosci 2005;28:325-33.

[42] Llinás RR, Ribary U, Jeanmonod D, Kronberg E, Mitra PP. Thalamocortical dysrhythmia: a neurological and neuropsychiatric syndrome characterized by magnetoencephalography. Proc Natl Acad Sci USA 1999;96:15222-7.

[43] Makin S. Imaging: show me where it hurts. Nature 2016;535:S8-9.

[44] Mano H, Kotecha G, Leibnitz K, Matsubara T, Sprenger C, Nakae A, Shenker N, Shibata M, Voon V, Yoshida W, Lee M, Yanagida T, Kawato M, Rosa MJ, Seymour B. Classification and characterisation of brain network changes in chronic back pain: a multicenter study. Wellcome Open Res 2018;3:19.

[45] Mansour A, Baria AT, Tetreault P, Vachon-Presseau E, Chang PC, Huang L, Apkarian AV, Baliki MN. Global disruption of degree rank order: a hallmark of chronic pain. Sci Rep 2016;6:34853.

[46] Maris E, Oostenveld R. Nonparametric statistical testing of EEG- and MEG-data. J Neurosci Methods 2007;164:177-90.

[47] May ES, Nickel MM, Ta Dinh S, Tiemann L, Heitmann H, Voth I, Tölle TR, Gross J, Ploner M. Prefrontal gamma oscillations reflect ongoing pain intensity in chronic back pain patients. Hum Brain Mapp 2019;40: 293-305.

[48] Melzack R. The short-form McGill pain questionnaire. PAIN 1987;30: $191-7$.

[49] Moriarty O, McGuire BE, Finn DP. The effect of pain on cognitive function: a review of clinical and preclinical research. Prog Neurobiol 2011;93: 385-404.

[50] Mouraux A, lannetti GD. The search for pain biomarkers in the human brain. Brain 2018;141:3290-307.

[51] Oostenveld R, Fries P, Maris E, Schoffelen JM. FieldTrip: open source software for advanced analysis of MEG, EEG, and invasive electrophysiological data. Comput Intell Neurosci 2011;2011:156869.

[52] Pinheiro ES, de Queirós FC, Montoya P, Santos CL, do Nascimento MA, Ito $\mathrm{CH}$, Silva M, Nunes Santos DB, Benevides S, Miranda JG, Sá KN, Baptista AF. Electroencephalographic patterns in chronic pain: a systematic review of the literature. PLoS One 2016;11:e0149085.

[53] Ploner M, Sorg C, Gross J. Brain rhythms of pain. Trends Cogn Sci 2017; 21:100-10.

[54] Polanía R, Nitsche MA, Ruff CC. Studying and modifying brain function with non-invasive brain stimulation. Nat Neurosci 2018;21:174-87.

[55] Rauschecker JP, May ES, Maudoux A, Ploner M. Frontostriatal gating of tinnitus and chronic pain. Trends Cogn Sci 2015;19:567-78.

[56] Reardon S. Neuroscience in court: the painful truth. Nature 2015;518: 474-6. 
[57] Rubinov M, Sporns O. Complex network measures of brain connectivity: uses and interpretations. Neuroimage 2010;52:1059-69.

[58] Sarnthein J, Stern J, Aufenberg C, Rousson V, Jeanmonod D. Increased EEG power and slowed dominant frequency in patients with neurogenic pain. Brain 2006;129:55-64.

[59] Schmidt S, Naranjo JR, Brenneisen C, Gundlach J, Schultz C, Kaube H, Hinterberger $T$, Jeanmonod D. Pain ratings, psychological functioning and quantitative EEG in a controlled study of chronic back pain patients. PLoS One 2012; 7:e31138.

[60] Schoffelen JM, Gross J. Source connectivity analysis with MEG and EEG. Hum Brain Mapp 2009;30:1857-65.

[61] Selim AJ, Rogers W, Fleishman JA, Qian SX, Fincke BG, Rothendler JA, Kazis LE. Updated U.S. Population standard for the Veterans RAND 12item health survey (VR-12). Qual Life Res 2009;18:43-52.

[62] Seminowicz DA, Moayedi M. The dorsolateral prefrontal cortex in acute and chronic pain. J Pain 2017;18:1027-35.

[63] Sitaram R, Ros T, Stoeckel L, Haller S, Scharnowski F, Lewis-Peacock J, Weiskopf N, Blefari ML, Rana M, Oblak E, Birbaumer N, Sulzer J. Closedloop brain training: the science of neurofeedback. Nat Rev Neurosci 2017;18:86-100.

[64] Smith SM, Dworkin RH, Turk DC, Baron R, Polydefkis M, Tracey I, Borsook D, Edwards RR, Harris RE, Wager TD, Arendt-Nielsen L, Burke LB, Carr DB, Chappell A, Farrar JT, Freeman R, Gilron I, Goli V, Haeussler J, Jensen T, Katz NP, Kent J, Kopecky EA, Lee DA, Maixner W, Markman JD, McArthur JC, McDermott MP, Parvathenani L, Raja SN, Rappaport BA, Rice ASC, Rowbotham MC, Tobias JK, Wasan AD, Witter J. The potential role of sensory testing, skin biopsy, and functional brain imaging as biomarkers in chronic pain clinical trials: IMMPACT considerations. J Pain 2017;18:757-77.

[65] Spielberger CD, Gorsuch RL, Lushene R, Vagg PR, Jacobs GA. Manual for the state-trait anxiety inventory. Palo Alto: Consulting Psychologists Press, 1983.

[66] Stam CJ, Nolte G, Daffertshofer A. Phase lag index: assessment of functional connectivity from multi channel EEG and MEG with diminished bias from common sources. Hum Brain Mapp 2007;28:1178-93.

[67] Stern J, Jeanmonod D, Sarnthein J. Persistent EEG overactivation in the cortical pain matrix of neurogenic pain patients. Neuroimage 2006;31:721-31.

[68] Thomson DJ. Spectrum estimation and harmonic analysis. Proc IEEE 1982;70:1055-96.

[69] Uhlhaas PJ, Singer W. Neural synchrony in brain disorders: relevance for cognitive dysfunctions and pathophysiology. Neuron 2006;52:155-68.

[70] Uhlhaas PJ, Singer W. Neuronal dynamics and neuropsychiatric disorders: toward a translational paradigm for dysfunctional large-scale networks. Neuron 2012;75:963-80.

[71] Upadhyay J, Geber C, Hargreaves R, Birklein F, Borsook D. A critical evaluation of validity and utility of translational imaging in pain and analgesia: utilizing functional imaging to enhance the process. Neurosci Biobehav Rev 2018;84:407-23.

[72] Vachon-Presseau E, Tetreault P, Petre B, Huang L, Berger SE, Torbey S, Baria AT, Mansour AR, Hashmi JA, Griffith JW, Comasco E, Schnitzer TJ, Baliki MN, Apkarian AV. Corticolimbic anatomical characteristics predetermine risk for chronic pain. Brain 2016;139:1958-70.

[73] van Diessen E, Numan T, van Dellen E, van der Kooi AW, Boersma M, Hofman D, van Lutterveld R, van Dijk BW, van Straaten EC, Hillebrand A, Stam CJ. Opportunities and methodological challenges in EEG and MEG resting state functional brain network research. Clin Neurophysiol 2015; 126:1468-81.

[74] Van Veen BD, van Drongelen W, Yuchtman M, Suzuki A. Localization of brain electrical activity via linearly constrained minimum variance spatial filtering. IEEE Trans Biomed Eng 1997;44:867-80.

[75] Vanneste S, Ost J, Van Havenbergh T, De Ridder D. Resting state electrical brain activity and connectivity in fibromyalgia. PLoS One 2017; 12:e0178516.

[76] Vanneste S, Song JJ, De Ridder D. Thalamocortical dysrhythmia detected by machine learning. Nat Commun 2018;9:1103.

[77] Velly AM, Mohit S. Epidemiology of pain and relation to psychiatric disorders. Prog Neuropsychopharmacol Biol Psychiatry 2018;87(pt B): 159-167.

[78] Vinck M, Oostenveld R, van Wingerden M, Battaglia F, Pennartz CM. An improved index of phase-synchronization for electrophysiological data in the presence of volume-conduction, noise and sample-size bias. Neuroimage 2011;55:1548-65.

[79] Vuckovic A, Hasan MA, Fraser M, Conway BA, Nasseroleslami B, Allan DB. Dynamic oscillatory signatures of central neuropathic pain in spinal cord injury. J Pain 2014;15:645-55.

[80] Wager TD, Atlas LY, Lindquist MA, Roy M, Woo CW, Kross E. An fMRIbased neurologic signature of physical pain. N Engl J Med 2013;368: 1388-97.

[81] Wang XJ. Neurophysiological and computational principles of cortical rhythms in cognition. Physiol Rev 2010;90:1195-268.

[82] Watts DJ, Strogatz SH. Collective dynamics of "small-world" networks. Nature 1998;393:440-2.

[83] Winkler I, Debener S, Müller KR, Tangermann M. On the influence of highpass filtering on ICA-based artifact reduction in EEG-ERP. Conf Proc IEEE Eng Med Biol Soc 2015;2015:4101-5.

[84] Woo CW, Chang LJ, Lindquist MA, Wager TD. Building better biomarkers: brain models in translational neuroimaging. Nat Neurosci 2017;20:365-77.

[85] Zhang Z, Gadotti VM, Chen L, Souza IA, Stemkowski PL, Zamponi GW. Role of prelimbic GABAergic circuits in sensory and emotional aspects of neuropathic pain. Cell Rep 2015;12:752-9. 